\title{
Alternative polyadenylation factors link cell cycle to migration
}

\author{
Mithun Mitra ${ }^{1,2}$, Elizabeth L Johnson ${ }^{3}$, Vinay S Swamy ${ }^{4}$, Lois E Nersesiann ${ }^{5}$, David C Corney ${ }^{1,3}$, David G Robinson ${ }^{6}$, \\ Daniel G Taylor ${ }^{1}$, Aaron M Ambrus ${ }^{1}$, David Jelinek', Wei Wang ${ }^{6}$, Sandra L Batista ${ }^{7}$ and Hilary A Coller ${ }^{1,2^{*}}$ (i)
}

\begin{abstract}
Background: In response to a wound, fibroblasts are activated to migrate toward the wound, to proliferate and to contribute to the wound healing process. We hypothesize that changes in pre-mRNA processing occurring as fibroblasts enter the proliferative cell cycle are also important for promoting their migration.

Results: RNA sequencing of fibroblasts induced into quiescence by contact inhibition reveals downregulation of genes involved in mRNA processing, including splicing and cleavage and polyadenylation factors. These genes also show differential exon use, especially increased intron retention in quiescent fibroblasts compared to proliferating fibroblasts. Mapping the $3^{\prime}$ ends of transcripts reveals that longer transcripts from distal polyadenylation sites are more prevalent in quiescent fibroblasts and are associated with increased expression and transcript stabilization based on genome-wide transcript decay analysis. Analysis of dermal excisional wounds in mice reveals that proliferating cells adjacent to wounds express higher levels of cleavage and polyadenylation factors than quiescent fibroblasts in unwounded skin. Quiescent fibroblasts contain reduced levels of the cleavage and polyadenylation factor CstF-64. CstF-64 knockdown recapitulates changes in isoform selection and gene expression associated with quiescence, and results in slower migration.
\end{abstract}

Conclusions: Our findings support cleavage and polyadenylation factors as a link between cellular proliferation state and migration.

Keywords: mRNA processing, Proliferation, Quiescence, Migration, Wound healing

\section{Background}

Fibroblasts within the dermis bear much of the responsibility for the secretion and maintenance of extracellular matrix proteins [1]. Fibroblasts in unwounded skin are mostly in a state of quiescence in which they have reversibly exited the proliferative cell cycle $[1-3]$. In the initial response to a wound, mitogens and chemokines such as platelet-derived growth factor and fibroblast growth factor released by platelets and keratinocytes stimulate fibroblasts to migrate to the wound-healing environment and proliferate [1-4]. In the wounded tissue, fibroblasts secrete collagen and other extracellular matrix molecules that remodel the extracellular environment and promote

\footnotetext{
*Correspondence: hcoller@ucla.edu

'Department of Molecular, Cell and Developmental Biology, University of California, Los Angeles, Los Angeles, CA, USA

2Department of Biological Chemistry, David Geffen School of Medicine,

University of California, Los Angeles, CA, USA

Full list of author information is available at the end of the article
}

the formation of a scar [3]. While fibroblasts are recognized to play an important role in normal skin and in the wound-healing environment, we do not yet have a full appreciation of the molecular mechanisms that control the changes in fibroblast behavior in the context of a wound.

We have been studying the transition between proliferation and quiescence in a model system in primary human dermal fibroblasts [5-9]. Using microarrays, we and others have shown that a shift between proliferation and quiescence is associated with a major reprogramming of gene expression patterns, and that these gene expression changes are important for the functional attributes of quiescent cells, such as their ability to re-enter the cell cycle [9-12]. Based on our previous studies showing changes in the levels of splicing factors as fibroblasts transition between proliferation and quiescence [9], and earlier studies showing that proliferating cells, stem cells, activated cells, and cancer cells rely heavily on alternative polyadenylation (APA) by preferential use of proximal polyadenylation 
sites [13-21], we sought to understand whether alternative isoform use [16, 22, 23] could represent a link between proliferation and migration.

To address this question, we defined the changes in isoform use and polyadenylation site selection that occur as cells transition from proliferation to quiescence. We found that APA factors are expressed at lower levels as fibroblasts become quiescent, and that knockdown of these factors results in APA and gene expression changes that overlap with the changes that occur with quiescence. Longer transcripts that end at distal polyadenylation sites tend to be more stable than shorter transcripts generated from proximal polyadenylation site use in proliferating cells. We also discovered that APA factors are functionally important for the transition to a more migratory state in proliferating versus quiescent fibroblasts and affect migration in cancer cells as well. Our data, taken as a whole, provide a deeper understanding of the role of mRNA processing in the close association between proliferation and migration.

\section{Results}

Entry into quiescence results in downregulation of genes involved in the cell cycle, mRNA processing, and motility Primary human dermal fibroblasts were isolated from human skin samples as previously described [24]. Fibroblasts isolated from two different donors were collected in proliferating conditions or after being induced into quiescence by 7 days of contact inhibition $(7 \mathrm{dCI})$ of proliferation [7]. RNA-Seq and microarray analyses were performed to determine changes in gene expression between three samples of proliferating and matched $7 \mathrm{dCI}$ cells (Fig. 1a and Additional file 1: Table S1) [25]. Among the 19,673 genes monitored, transcripts from 1993 genes (10.1\%) changed in expression twofold or more, demonstrating widespread changes in gene expression with contact inhibition-induced quiescence (Fig. 1b). Expression levels for $52 \%$ of these genes were upregulated in $7 \mathrm{dCI}$ compared with proliferating fibroblasts, and $48 \%$ were downregulated in $7 \mathrm{dCI}$ fibroblasts.

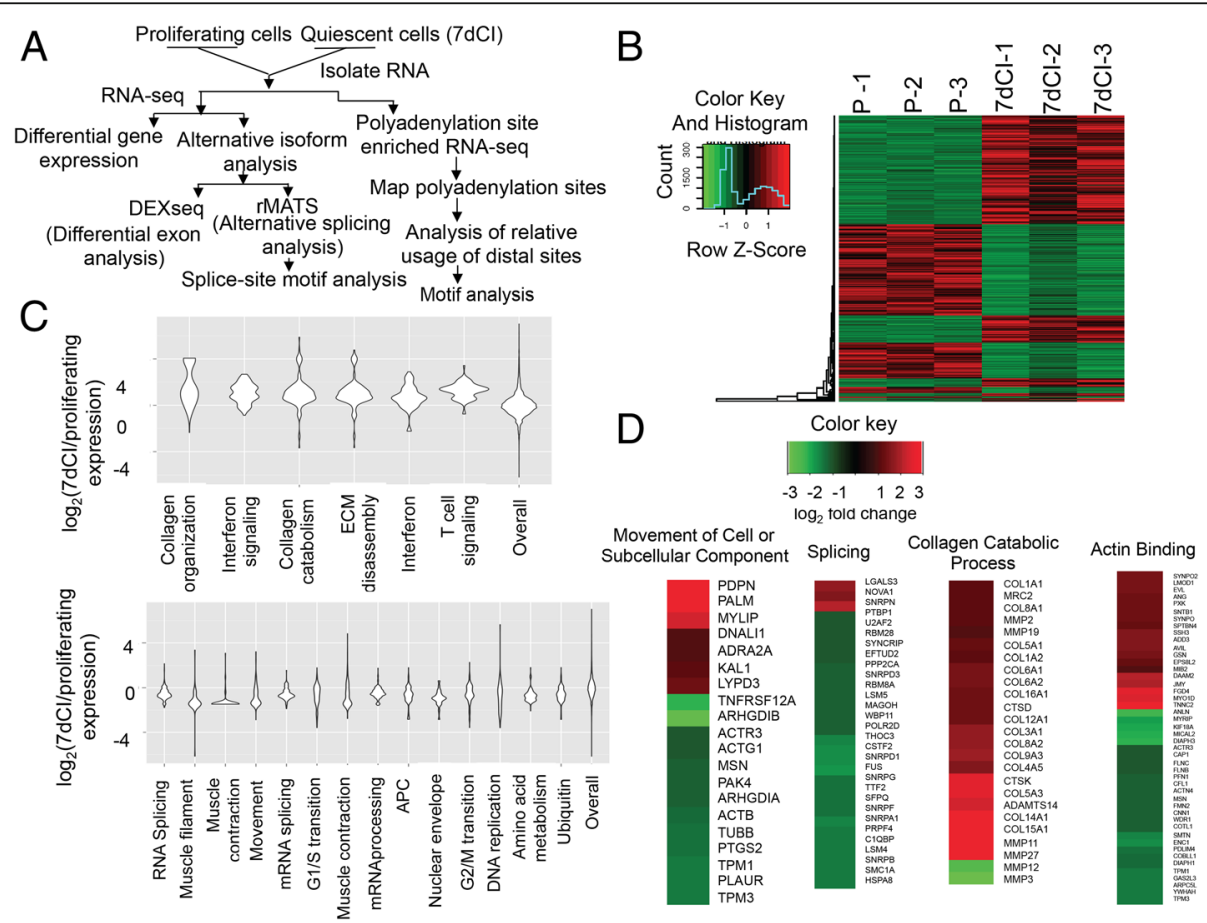

Fig. 1 RNA-Seq analysis of gene expression changes in proliferating versus quiescent fibroblasts. a Schematic of RNA-Seq-based analysis of proliferating and quiescent fibroblasts performed in this study. $\mathbf{b}$ Total RNA was isolated from three independent biological replicates of proliferating fibroblasts and three matched independent biological replicates of $7 \mathrm{dCl}$ fibroblasts. RNA samples were converted to cDNA libraries and sequenced on an Illumina Hi-Seq 2000. Reads were aligned to the human genome (hg19 human reference sequence) and the number of reads mapping to each gene (UCSC gene annotation) in the genome was determined. A heatmap of read counts for 1993 genes with at least a twofold change in expression and a false discovery rate (FDR) $<5 \%$ is shown. Hierarchical clustering is denoted by the dendrogram to the left of the heatmap. A color key and a histogram displaying the density of genes at a given color intensity are shown in the upper left corner. c Gene set enrichment analysis was used to determine the gene sets most significantly upregulated (top) or downregulated (bottom) with quiescence. Gene sets are listed in descending order of statistical significance from left to right. A histogram of the $\log _{2}$ (fold-change) of the normalized read count in $7 \mathrm{dCl}$ compared to proliferating fibroblasts for each gene in the gene set is plotted in a violin plot representation. $\mathbf{d}$ Heat maps of genes within selected gene set enrichment categories are provided. The $\log _{2}$ ratio of normalized RNA-Seq counts in $7 \mathrm{dCl}$ compared with proliferating fibroblasts are shown. Red indicates higher expression in quiescent than proliferating fibroblasts; green indicates higher expression in proliferating than quiescent fibroblasts. Only genes in each category that change in expression two-fold or more are included 
Correlation between biological replicates analyzed by RNA-Seq was high $\left(R^{2}\right.$ values greater than or equal to 0.83$)$ (Additional file 1: Figure S1A). When the same samples were analyzed with microarrays, the differential gene expression detected by microarray was largely in agreement with that detected by RNA-Seq $\left(r^{2}=0.785, p<0.001\right)$ (Additional file 1: Figure S1B). Further, gene expression changes detected by RNA-Seq correlated well with the previously published "quiescence program" of gene expression changes identified in fibroblasts induced into quiescence by multiple independent conditions [9] (Additional file 1: Figure S1C). The findings support previous studies showing that quiescence is associated with regulation of a significant fraction of the genome $[9,10,26]$.

Gene set enrichment analysis (GSEA) [27, 28] revealed that expression of genes involved in DNA replication and cell cycle regulation was downregulated in $7 \mathrm{dCI}$ compared with proliferating fibroblasts (Fig. 1c), consistent with cell cycle exit in contact-inhibited conditions. Expression of genes associated with extracellular matrix remodeling and collagen metabolism was upregulated with quiescence (Fig. 1c, d), consistent with our previous findings [6, 7]. Indeed, COL21A1, a collagen found associated with collagen I, is among the genes most strongly induced in quiescent compared with proliferating fibroblasts (Additional file 1: Table S2). Expression of genes in the categories of muscle filament sliding, regulation of muscle contraction, movement, and muscle contraction was downregulated in contact-inhibited compared with proliferating fibroblasts (Fig. 1c, d). Four genes involved in cell motility were among the most strongly downregulated genes with quiescence (KISS1, ACTC1, PODXL, and RLTPR) (Table 1 and Additional file 1: Table S2). Thus, we found that proliferating fibroblasts express higher levels of transcripts associated with motility and cytoskeletal remodeling.

Transcripts associated with splicing and polyadenylation were mostly downregulated in $7 \mathrm{dCI}$ compared with proliferating fibroblasts (Fig. 1c, d), consistent with previous reports $[9,21]$. Transcripts encoding many of the proteins that are considered core components of the spliceosome were slightly downregulated in contactinhibited compared with proliferating fibroblasts (Additional file 1: Table S3), with three genes reaching statistical significance (U1C (2.26-fold reduction), PRPF4 (2.77-fold reduction), and PPIH (2.89-fold reduction)). Expression levels of cleavage and polyadenylation factors were also reduced with quiescence (Additional file 2). We hypothesized that in addition to changes in gene expression, alterations in mRNA processing events between proliferating and quiescent fibroblasts could also contribute to functional changes in quiescent and proliferating states.

\section{Quiescent fibroblasts retain more exons and introns than proliferating fibroblasts}

To better understand changes in mRNA processing associated with proliferation, we investigated our RNA-Seq data further to identify examples of alternative start site, alternative splicing, or alternative polyadenylation. Applying the DEXSeq algorithm [29], we discovered 1975 exons, encoded within 1218 genes, with differential expression between proliferating and $7 \mathrm{dCI}$ fibroblasts (Additional file 3). Using g:Profiler [30], we found that genes that undergo alternative isoform expression in proliferating versus quiescent cells are enriched in categories of RNA binding, RNA processing, translational elongation, and RNA splicing (Table 2, Additional file 4). Thus, genes involved in RNA processing are themselves particularly likely to be alternatively processed during the transition between proliferation and quiescence.

To better understand the frequency of specific types of splicing events that occurred differentially in proliferating and quiescent fibroblasts, we applied the rMATS computational algorithm [31-33] (Fig. 2a, Additional file 5). Skipped exons (exons that are present in proliferating, but

Table 1 List of genes involved in motility that are altered with quiescence

\begin{tabular}{|c|c|}
\hline Type of change (analysis) & Genes \\
\hline Differential expression (DESeq) & $\begin{array}{l}\text { KISS1, ACTC1, PODXL, RLTPR, PDPN, PALM, MYLIP, DNALI1, ADRA2A, KAL1, LYPD3, TNFRSF12A, } \\
\text { ARHGDIB, ACTR3, ACTG1, MSN, PAK4, ARHGDIA, ACTB, TUBB, PTGS2, TPM1, PLAUR, TPM3 }\end{array}$ \\
\hline Alternative splicing (rMATS) & $\begin{array}{l}\text { FYN, HGF, CD44, MAP4, ATP2B4, MYLK, ACTN1, SCARB1, FGFR1, FAT1, MYL6, TSPO, AKT2, BRAT1, } \\
\text { ARHGAP21, GTPBP4, SOD2, FN1, WIPF1, CD58, KIF17, ITGB1BP1, GLIPR2, CALD1, LRP1, KLC1, KTN1 } \\
\text { CHN1, POSTN, NUMB, ENPP2, KIF13A, TPM1, CLTC, MINK1, SH3KBP1, CACNA1C, DAB2, THY1, NCK } \\
\text { SHC1, NFASC, IL17RC, LAMB2, PTPRM, GAS6, CCDC125, LAMA2, FLNA, SPTAN1, TPM2, CDC42BPB }\end{array}$ \\
\hline $\begin{array}{l}\text { UTR-type alternative polyadenylation for } 2 \mathrm{pA} \\
\text { site-containing genes }\end{array}$ & $\begin{array}{l}\text { MACF1, LMNA, DDR2, LAMC1, NAV1, PEX13, ACTR2, IL1R1, MYO1B, SP100, TRAK1, NCK1, OPA1, } \\
\text { PDGFRA, AP3S1, TUBB, PTP4A1, FGFR1OP, ANLN, GLIPR2, AMOTL1, PTPN11, MMP14, FRMD6, } \\
\text { SMAD3, DYNLL2, PTPRA, DOK5, COL18A1, SSX2IP, NRAS, CERS2, SDC1, DPP4, DYNC1LI1, MAP4, } \\
\text { WNT5A, CD47, DPYSL3, LAMA4, ARHGAP18, CITED2, GNA12, STC1, EXT1, AP3M1, BLOC1S2, } \\
\text { RRAS2, TBX5, SYNJ2BP, EMP2, AMFR, STAT3, COL1A1, CCBE1, AP3D1, SDC4, APP, RBFOX2, } \\
\text { MYH9, RAP2C }\end{array}$ \\
\hline
\end{tabular}


Table $\mathbf{2}$ List of splicing genes undergoing differential pre-mRNA processing with quiescence

\begin{tabular}{ll}
\hline Type of pre-mRNA processing (analysis) & Splicing genes \\
\hline Differential exon use (DEXSeq) & KDM1A, LUC7L, AQR, ZCCHC8, SFSWAP, U2AF2, SUGP2, SNRPA, YTHDC1, FUS, PAPOLA, HNRNPC, \\
& HNRNPH3, HNRNPM, POLR2E, SNU13, RBFOX2, SRSF5, ACIN1, PABPC1L, USB1, SNRNP70, RBM28, \\
& LSM5, CASC3, LUC7L3, DHX15, MAGOHB, SRSF9, SRSF3, PAPOLG, SF3B1, SRSF7, SRSF4, SFPQ, \\
& SRSF11, RBM25, DDX39A, SRSF6, RBM39, SNRPA1, ZRANB2, SRRM1, RBM17, CCNH, RBMX2, TRA2B, \\
& HNRNPD, SCAF11, SNRPG, SNRNP200, SREK1, RNF20, CCAR2, U2AF1, CHTOP, ZNF326, TRA2A, \\
& CDK12, SF1, HNRNPH1, RBM4B, RSRC1, GEMIN4, NPM1, SF3A3, PRPF39, ADARB1, UBL5 \\
& LUC7L, ZCCHC8, SRRT, HNRNPC, HNRNPH3, NHP2L1, DDX17, RBM23, PRMT5, SRSF5, ACIN1, \\
Alternative splicing (rMATS) & PQBP1, SNRNP70, POLR2I, DDX5, MAGOHB, SRSF3, PRPF4B, SF3B1, SRSF7, TIA1, SRSF11, RBM25, \\
& DDX39A, SRSF6, SNRPB, DNAJC8, RBM39, SNRPA1, SRRM1, PNPT1, HNRNPD, SCAF11, MBNL1, \\
& SREK1, AFF2, RBPMS, U2AF1, CHTOP, SRSF2, TRA2A, HNRNPH1, PRPF39, UBL5, DDX39B \\
UTR-type alternative polyadenylation for 2pA & WDR77, SNRNP40, SF3A3, CHTOP, TSEN15, KHDRBS1, RBM17, HNRNPH3, CSTF3, RBM4B, \\
site-containing genes & MAGOHB, SART3, CMTR2, TXNL4B, POLR2C, SF3B3, SRSF2, RNMT, GTF2F1, KHSRP, HNRNPUL1, \\
& PTBP1, CIR1, TIA1, GCFC2, APP, RBFOX2, NHP2L1, POLDIP3, ISY1, NCBP2, RSRC1, POLR2H, PAPD4 \\
Upstream region-type alternative polyadenylation & HNRNPR, WBP11, RBBP6, SAFB2, PRPF4B, PSIP1, ZRSR2 \\
for 2 pA site-containing genes & \\
\hline
\end{tabular}

not quiescent, cells or vice versa) were the most common type of event detected (319 events, $53 \%$ of events). Of the splicing events detected by rMATS, 39\% were also detected by DEXSeq. More exons were preferentially included in quiescent compared with proliferating conditions, than proliferating compared with quiescent conditions (1.5-fold, Fisher's exact test, two-tailed $p$ value $=0.013$ ) (Fig. 2a). These exon-switching events provide opportunities for regulation of protein function based on the inclusion or exclusion of individual exons. Introns were significantly more frequently retained in quiescent than proliferating fibroblasts (3.7-fold, Fisher's exact test, two-tailed $p$ value $<0.0001$ ) (Fig. 2a). $8.2 \%$ of the transcripts associated with retained intron events are annotated as nonsense-mediated decay (NMD) candidates (18 unique NMD transcripts/220 total unique intron retention transcripts in the Ensembl database). Gene ontology (GO) analysis of the differentially spliced genes revealed that genes that undergo alternative splicing with quiescence are enriched for the categories of RNA binding, RNA processing, and RNA splicing (Table 2 and Additional file 6), consistent with a growing literature demonstrating that genes involved in mRNA splicing are themselves regulated by splicing events [30, 34-37].

\section{Some auxiliary splicing factors are downregulated in quiescent fibroblasts}

To understand the changes in splicing in quiescent compared with proliferating fibroblasts, we investigated changes in the expression of splicing factors. Our RNA-Seq data revealed that expression from RNA splicing genes is modestly downregulated in contact-inhibited fibroblasts (Fig. 1c, d and Additional file 1: Table S3). We monitored protein levels of splicing factors with immunoblotting in fibroblasts that were proliferating or induced into quiescence by 7 days of contact inhibition $(7 \mathrm{dCI})$ or by serum starvation (7dSS). Levels of essential splicing factor U2AF65 were similar in proliferating and quiescent fibroblasts. Levels of core factor
U1-70K and auxiliary factors TRA2 $\beta$ and FUS were downregulated in quiescent compared with contact-inhibited fibroblasts (Fig. 2b). Lower levels of some splicing factors in quiescent fibroblasts may contribute to the increased intron retention in quiescent conditions [38, 39].

\section{Weaker splice sites for retained introns}

In addition to lower levels of splicing factors, intron retention has been associated with weak splice sites [40, 41]. To better understand why some introns are retained in proliferating or quiescent cells, we analyzed the extent to which 5 ' splice sites (9-nt length) and 3' splice sites (23 nt) of differentially retained introns match consensus splice sites [42]. We determined the probability of observing each sequence given the position weight matrix for consensus splice sites. Sequences at splice sites for introns differentially retained in proliferating or quiescent states matched the consensus sequence less well than the sequences near constitutively spliced exons, with a strong effect at the $3^{\prime}$ splice site (Fig. 2c). These findings are consistent with previous studies that also showed that 3' splice sites are enriched for C's compared with T's in the polypyrimidine tracts of introns that are retained [43]. Thus, in proliferating fibroblasts that have higher levels of most splicing factors, intron retention may be especially sensitive to the 3 ' splice sequence.

\section{A shift toward the use of more distal polyadenylation sites in quiescence}

A shift toward the use of distal polyadenylation sites has been observed in previous studies that showed that non-dividing cells [21] and differentiated cells [18, 20, $44,45]$ predominantly use distal polyadenylation sites, while proliferating cells $[18,21]$ and cancer cell lines $[20$, $45,46]$ tend to use proximal polyadenylation sites. Our DEXSeq analysis revealed that many of the changes in isoform expression detected between proliferating and $7 \mathrm{dCI}$ fibroblasts involve the last exon of the analyzed 
A

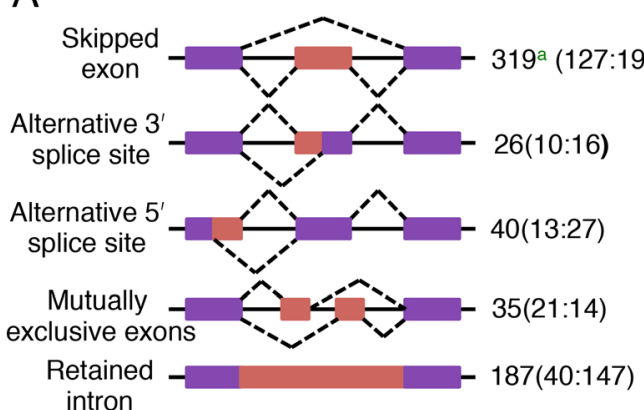

aNumber of splicing events

bEvents with higher inclusion level in proliferating cells : events with higher inclusion level in quiescent cells

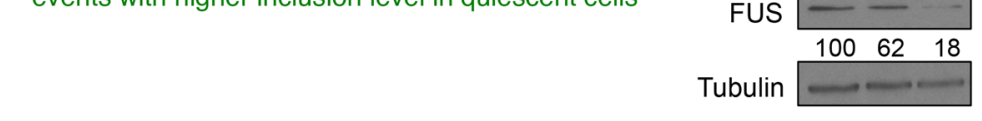

C
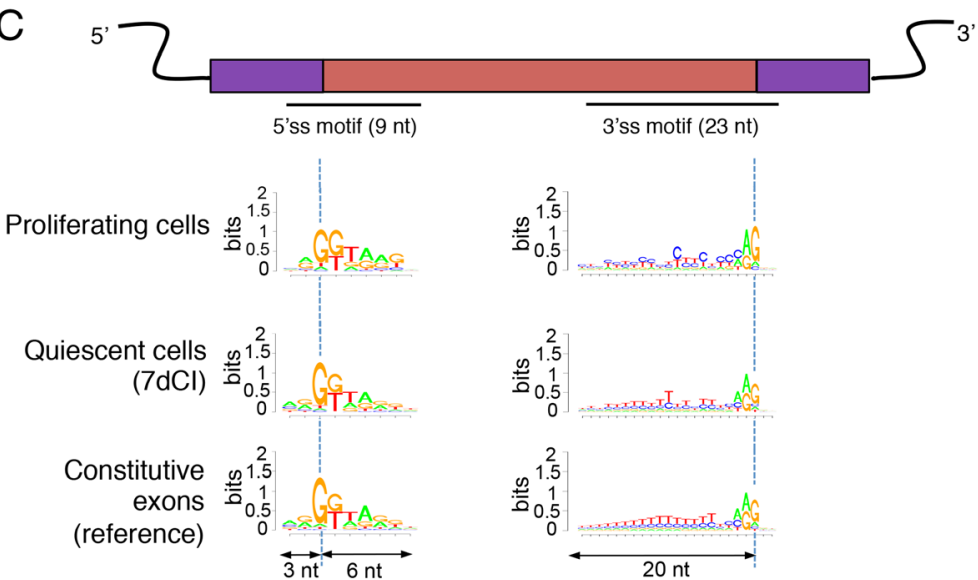

B

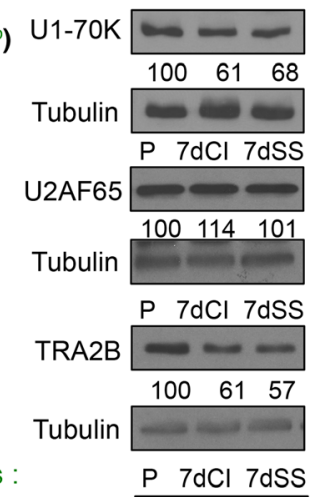

FUS

$$
\text { ass }
$$

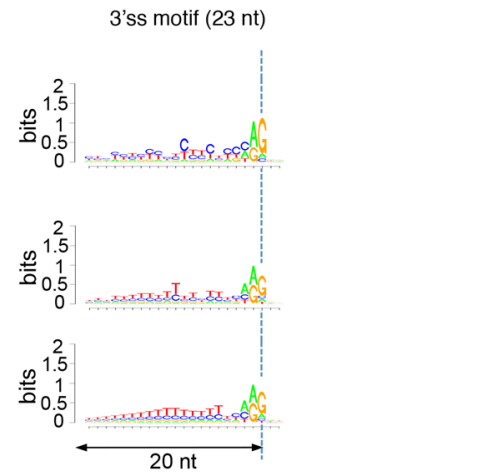

Fig. 2 Differential splicing in proliferating and quiescent fibroblasts. a rMATS was applied to RNA-Seq data from three biological replicates of proliferating fibroblasts and three biological replicates of contact-inhibited fibroblasts. Splicing events with an FDR $<0.05$ are shown. The total numbers of splicing events are reported. In parentheses, the number of events with higher inclusion in proliferating fibroblasts is provided, followed by the number of events with higher inclusion in quiescent fibroblasts. Skipped exons were significantly more likely to be included in quiescent fibroblasts (Fisher's exact test, two-tailed $p$ value $=0.013$ ). Introns were significantly more likely to be retained in quiescent fibroblasts (Fisher's exact test, two-tailed $p$ value $<0.0001$ ). $\mathbf{b}$ Immunoblotting of splicing factors in proliferating and quiescent fibroblasts. Levels of core splicing factor U2AF65 were similar in proliferating and quiescent fibroblasts. U1-70 K and auxiliary factors TRA2 $\beta$ and FUS were expressed at lower levels in $7 \mathrm{dCl}$ and $7 \mathrm{dSS}$ compared with proliferating fibroblasts. a-Tubulin was analyzed as a loading control. The ratio of splicing factor to tubulin, normalized to proliferating cells, is shown below. c Sequence logos [120] are provided for 5' and 3' sequences for exons that are constitutively spliced, and introns that are preferentially retained in proliferating or quiescent cells. The $y$-axis indicates bits of information [121]. $3^{\prime}$ splice site sequences were different between proliferating versus constitutive conditions ( $p$ value $<0.01$ for constitutive versus retained in proliferating conditions, ANOVA with Tukey's multiple comparison test) and quiescent versus constitutive conditions ( $p$ value $<0.01$ for constitutive versus retained in quiescent conditions)

transcript and would result in a change in polyadenylation site. For example, Inverted Formin, $\mathrm{FH} 2$ and $\mathrm{WH} 2$ domain (INF2), and brother of CDO (BOC) (Fig. 3a) exhibit alternative use of terminal exons in proliferating and $7 \mathrm{dCI}$ fibroblasts. Real-time PCR with isoform-specific primers confirmed that for both INF2 and BOC, the transition to quiescence in response to either $7 \mathrm{dCI}$ or $7 \mathrm{dSS}$ resulted in a change in polyadenylation site selection (Fig. 3b). For INF2, the strongest effect was a decrease in the use of the proximal polyadenylation site. For BOC, the strongest effect was an increase in the use of the distal polyadenylation site in quiescent fibroblasts. Restimulation of $7 \mathrm{dCI}$ fibroblasts to a proliferative state resulted in a reversal back toward a polyadenylation site selection profile more similar to that in proliferating cells for both INF2 and BOC.

To generate a large-scale dataset that would clearly define the 3' ends of transcripts in proliferating and quiescent $(7 \mathrm{dCI})$ fibroblasts, we applied polyadenylation site-enriched RNA-Seq [47]. With polyadenylation site-enriched RNA-Seq, 64\% of all mapped sequencing 


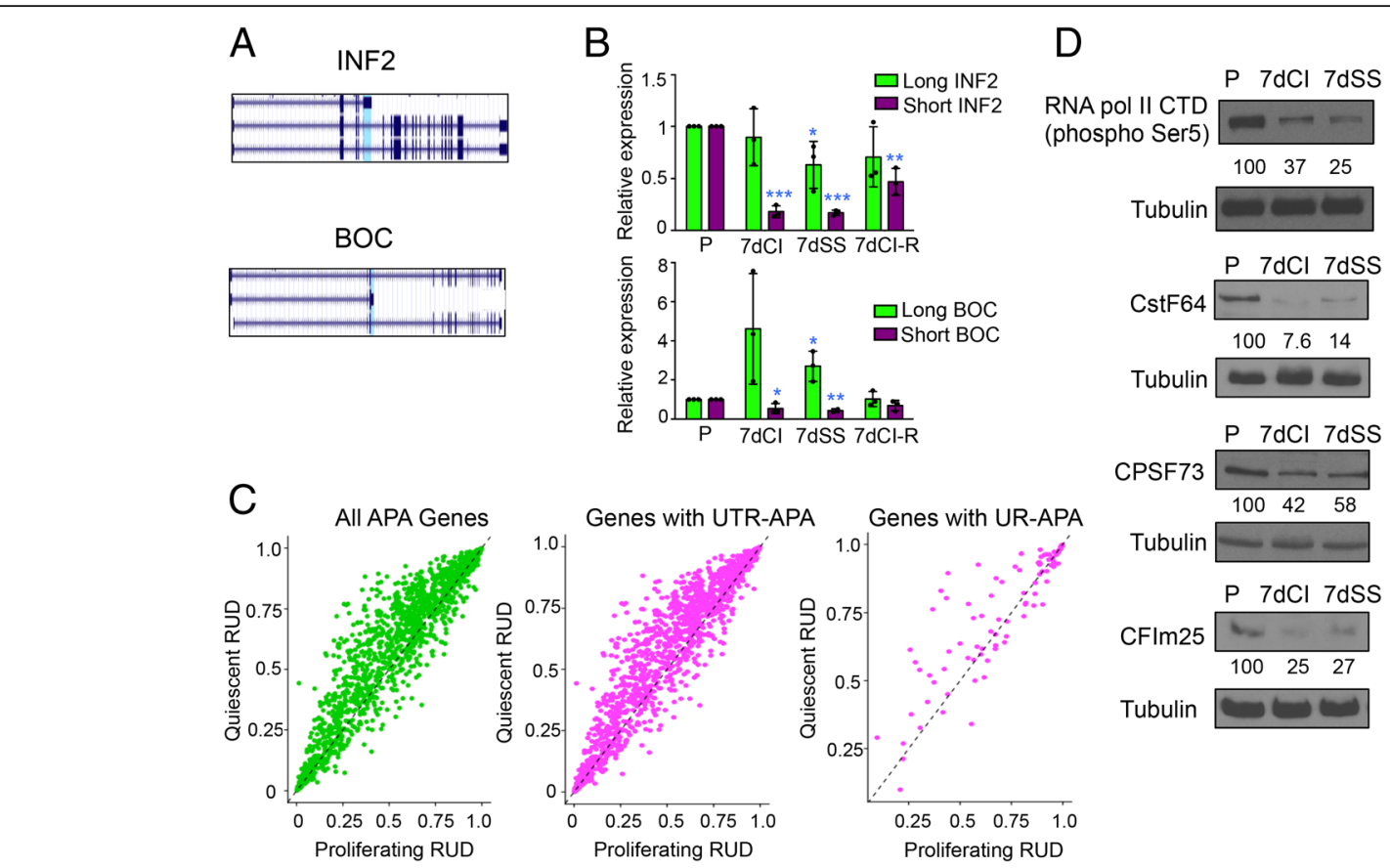

D

Fig. 3 Use of distal polyadenylation sites and lower levels of cleavage and polyadenylation factors during quiescence. a UCSC Genome browser views showing the long and short isoforms of INF2 and BOC. The differentially expressed exon is highlighted in cyan. $\mathbf{b}$ Real-time PCR validation of APA with quiescence. CDNA samples generated from fibroblasts that were proliferating, quiescent by contact inhibition or serum starvation, or induced into quiescence by serum-starvation and then restimulated, were analyzed with real-time PCR. Primers were designed to recognize the short (terminating at the proximal polyadenylation site) or long (terminating at the distal polyadenylation site) isoforms of INF2 or BOC.

Transitioning fibroblasts into quiescence resulted in reduced expression of the short isoform of INF2 and increased expression of the long isoform of BOC. Restimulating quiescent fibroblasts resulted in expression patterns of the short and long isoforms that more closely resemble proliferating cells. Plots show individual datapoints as dots. Bar graphs represent mean and average \pm S.D. The number of replicates for all conditions for short and long INF2 is 3 . The number of replicates for all conditions for long BOC is 3 . The number of replicates for $\mathrm{P}, 7 \mathrm{dCl}$, and $7 \mathrm{dCl}-\mathrm{R}$ for short $\mathrm{BOC}$ is 3. The number of replicates for 7dSS for short BOC is 2. Statistical significance in knockdown cells compared to control cells was determined for long and short isoforms with two-tailed, unpaired $t$ tests. For all figures, one asterisk indicates $p$ value $<0.05$. Two asterisks indicate $p$ value $<0.01$. Three asterisks indicate $p$ value $<0.001$. c A shift toward expression of longer isoforms in quiescent fibroblasts. Proliferating and $7 \mathrm{dCl}$ were analyzed by polyadenylation site-enriched RNA-Seq. Relative use of the distal polyadenylation site (RUD) for individual genes in proliferating fibroblasts is plotted on the $x$-axis and RUD for the same gene in quiescent conditions is plotted on the $y$-axis. The dashed black line indicates $y$ $=x$. The first plot (left) displays all genes with two detected polyadenylation sites. The middle plot displays UTR APA genes and the final plot (right) shows the same data for genes that undergo UR APA. d Immunoblotting was performed on protein lysates collected from proliferating, 7dCl and 7dSS fibroblasts for CstF-64, CFIm25, and CPSF73. Phosphorylation of serine 5 on RNA pol II CTD was monitored by immunoblotting and levels decline with quiescence. a-Tubulin was monitored as a loading control

reads matched a polyadenylation site (Additional file 1: Table S4). Polyadenylation site-enriched RNA-Seq data were used to determine the relative use of the distal (RUD) (reads mapping to the distal polyadenylation site/ total reads from proximal and distal polyadenylation sites) for each gene in proliferating and $7 \mathrm{dCI}$ conditions for detected genes with two polyadenylation sites (Additional file 7). For genes with greater than two polyadenylation sites (Additional file 8), a more general parameter called relative site usage (reads mapping to a polyadenylation site/total reads from all polyadenylation sites) was used. Data were highly reproducible when different biological replicates of proliferating and $7 \mathrm{dCI}$ samples were compared (Additional file 1: Figure S2A). Using polyadenylation site-enriched RNA-Seq, we confirmed the previous finding [21] of a shift toward the use of more distal polyadenylation sites upon entry into the quiescent state through contact inhibition (Fig. 3c, Additional file 7). Eighty-eight percent (628 out of 714) of genes with two polyadenylation sites, and with significant changes $(|R U D|>0.05)$ in alternative polyadenylation (APA) between the two cell states, were longer (greater use of distal pA sites compared to proximal pA sites) in the quiescent compared with the proliferating fibroblasts. For 572 of these 628 genes (91\%), the proximal polyadenylation site localizes to the 3 ' untranslated region (UTR; termed as UTR APA) (Fig. 3c), while for the remaining $9 \%$ of genes, the proximal polyadenylation site is found in the region upstream of the 3' UTR (upstream region (UR) APA) including introns and exons. Genes with two polyadenylation sites that undergo APA with quiescence were enriched in genes involved in RNA 
splicing and processing (Table 2 and Additional file 9). Genes that undergo APA with quiescence also included genes involved in cell migration (Table 1).

\section{Reduced levels of mRNA processing factors in quiescent fibroblasts}

To better understand the regulation of polyadenylation site use with quiescence, we monitored the levels of APA factors in proliferating and quiescent fibroblasts. Cleavage and polyadenylation of pre-mRNA transcripts are mediated by the coordinated activity of three core protein complexes [16]. The cleavage and polyadenylation specificity factor (CPSF) complex recognizes a hexameric sequence (AAUAAA or a similar sequence) in a 50-nt region upstream of the cleavage site [48, 49]; the $3^{\prime}$ pre-RNA, subunit 2, $64 \mathrm{kDa}$ (CSTF2 or CstF-64) subunit of the CstF complex recognizes a U-rich or G/U-rich region about 20-40 nucleotides downstream of the cleavage site [19, 50-53]; and Nudix (nucleoside diphosphate linked moiety X)-type motif 21 (NUDT21 or CFIm25) recognizes UGUA sequences upstream of the cleavage and polyadenylation sites [54]. CPSF73, a component of the CPSF complex, is the endonuclease that performs the cleavage event at the hexameric sequence [55]. Increased levels of CSTF complex proteins have been associated with the use of proximal polyadenylation sites $[19,56,57]$, while the CFIm complex has been reported to repress the use of proximal polyadenylation sites $[45,57,58]$. Our RNA-Seq data revealed that most of the core polyadenylation factors and auxiliary factors associated with cleavage and polyadenylation are modestly downregulated at the transcript level in quiescent compared with proliferating fibroblasts (Additional file 2). Among the core factors, CstF-64/ CSTF2 is strongly and significantly (3.1-fold) downregulated at the transcript level. Using immunoblotting, we found that the protein levels of CstF-64, CPSF73, and CFIm 25 are lower in $7 \mathrm{dCI}$ or $7 \mathrm{dSS}$ than in proliferating fibroblasts (Fig. 3d). By monitoring the extent of Serine 5 phosphorylation of RNA pol II carboxyterminal domain (CTD) as an indication of transcription initiation rate [59] with immunoblotting, we found that CstF-64 downregulation at the protein level with quiescence was stronger than the reduction in transcription initiation (Fig. 3d).

\section{Knockdown of cleavage and polyadenylation factors replicates polyadenylation site selection with quiescence} To better understand the role of cleavage and polyadenylation factors in polyadenylation site selection with quiescence, we introduced siRNAs that target CstF-64, CPSF73 or CFIm25, or a control siRNA, into fibroblasts. Strong knockdown of the targeted gene was confirmed with real-time PCR (Additional file 1: Figure S3). In comparison to control cells, knockdown of these polyadenylation factors did not significantly affect cell viability
(Additional file 1: Figure S4A and B). We tested whether knocking down the expression of cleavage and polyadenylation factors results in changes in the levels of shorter and longer isoforms of genes that undergo APA with quiescence using real-time PCR primers designed to recognize the short or long isoforms of INF2 or BOC (Fig. 3a). For INF2, knockdown of CstF-64 or CPSF73, but not CFIm25, resulted in reduced levels of the short isoform of INF2 and an increase in the long isoform of INF2 (Fig. 4a). For BOC, knockdown of CstF-64 or CPSF73, but not CFIm25, resulted in lower levels of the short BOC isoform (Fig. 4a). Knockdown of CstF-64 resulted in an increase in the long isoform of BOC (Fig. 4a).

To monitor global APA changes, we performed polyadenylation site-enriched RNA-Seq of fibroblasts transfected with a control siRNA or an siRNA that targets a polyadenylation factor (CstF-64, CPSF73, or CFIm25) [47]. Knockdown in two different strains of fibroblasts resulted in highly reproducible results (Additional file 1: Figure S2B). Each knockdown resulted in significant changes $(|\mathrm{RUD}|>0.05)$ in polyadenylation site selection, with CFIm 25 knockdown resulting in a clear shift toward use of more proximal polyadenylation sites (Additional file 1: Figure S4C and Additional file 10), consistent with previous reports $[60,61]$. We compared the genes that shift polyadenylation site use with quiescence with the results of knockdown of each cleavage and polyadenylation factor (Fig. 4b and Additional file 1: Figure S5A and $\mathrm{B})$. Among the three polyadenylation factors, knockdown of CFIm 25 resulted in the largest number of genes that shift to greater use of the proximal polyadenylation site (shorter isoforms), and the most genes that overlap with shifts to more proximal polyadenylation sites with quiescence (Fig. 4b and Additional file 1: Figure S5A). We observed significant overlap among the genes that use more distal polyadenylation sites (shift to longer isoforms) with quiescence and genes that use more distal polyadenylation sites with knockdown of each factor, with larger numbers of genes affected for CstF-64 or CPSF73 knockdown (Fig. 4b and Additional file 1: Figure S5A). Some of these changes in polyadenylation site use were specific for one factor, while some were regulated by more than one or even all three factors (Additional file 1: Figure S5B). For 626 unique genes that shift to distal polyadenylation site use with quiescence, 226 genes (36\%) also shift to distal polyadenylation site use with knockdown of one or more polyadenylation factors. For 86 genes that shift to proximal polyadenylation site use with quiescence, 38 (44\%) also shift to proximal polyadenylation site use with knockdown of one or more polyadenylation factors (Additional file 1: Figure S5B). 

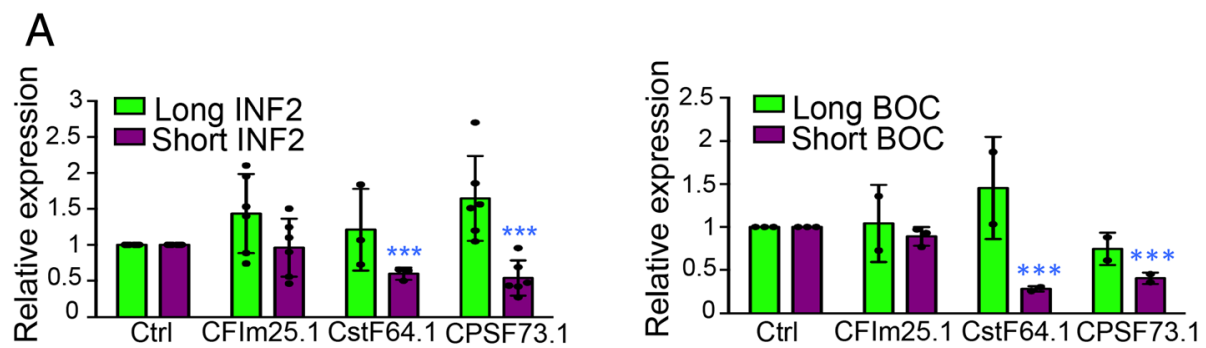

\section{B Genes with proximal $p A$ site use upon quiescence or KD}

Genes with distal $p A$ site use upon quiescence or KD
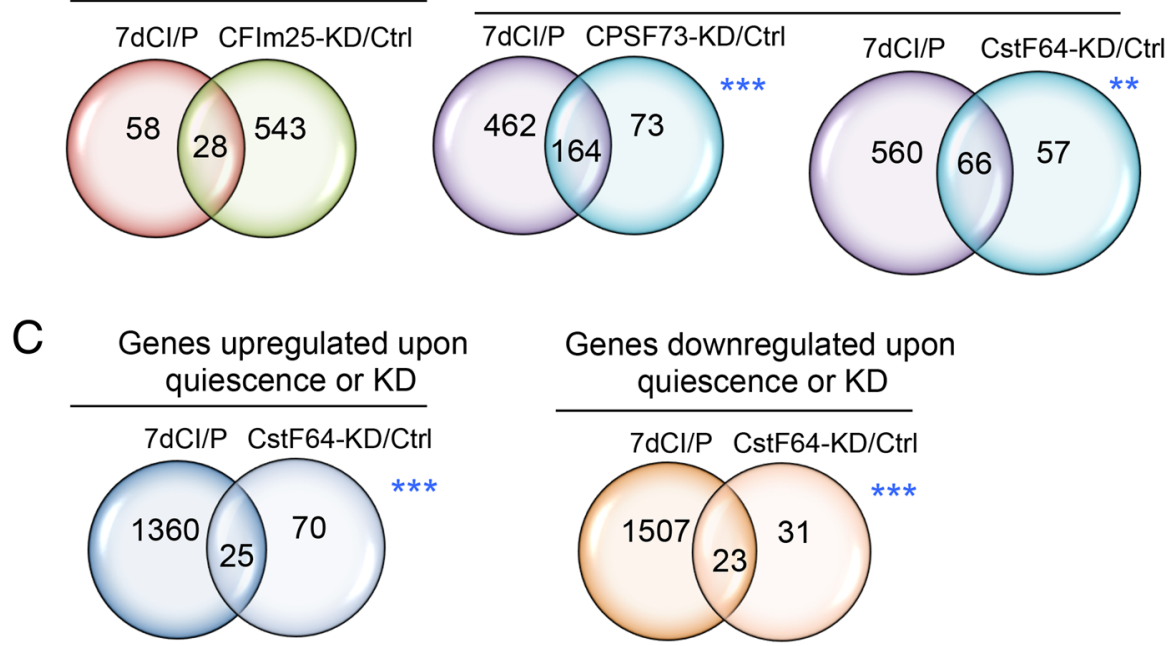

Fig. 4 Knockdown of cleavage and polyadenylation factors results in changes in isoform use and gene expression that overlap with quiescence. a Knockdown of cleavage and polyadenylation factors induces a shift in isoform expression. Real-time PCR was performed for the short and long isoforms of INF2 and BOC in proliferating fibroblasts expressing a control siRNA or an siRNA that targets CFIm25, CstF-64, or CPSF73. The short isoform of INF2 or BOC was significantly reduced in cells transfected with an siRNA against CstF64 or CPSF73. Plots show individual datapoints as dots. Bar graphs represent mean and average \pm S.D. The number of replicates for control, CFIm25 and CPSF73 knockdown for short and long INF2 is 6 . The number of replicates for CstF64 knockdown for short and long INF2 is 3 . The number of replicates for all conditions for long BOC is 2, except the control, which had 3 replicates. The number of replicates for control and CFIm25 knockdown for short BOC is 3 . The number of replicates for CstF64 and CPSF73 knockdown for short BOC is 2. Statistical significance in knockdown cells compared to control cells was determined for long and short isoforms with two-tailed, unpaired t-tests. b Overlap among genes that undergo APA with quiescence and knockdown of cleavage and polyadenylation factors. The overlap between genes that use the proximal polyadenylation site with quiescence and use a proximal polyadenylation site preferentially with CFIm25 knockdown is shown on the left. Overlap between genes that use distal polyadenylation sites with quiescence and genes that use distal polyadenylation sites with CPSF73 or CstF64 knockdown are shown in the middle and the right, respectively. c Overlap between genes upregulated with quiescence and genes upregulated with CstF-64 knockdown (left) and overlap between genes downregulated with quiescence and genes downregulated with CstF-64 knockdown (right). The overlap between groups of genes was tested using the hypergeometric test

Knockdown of CstF-64 resulted in changes in gene expression that significantly overlap with gene expression changes with quiescence (Fig. 4c and Additional file 11). Gene expression changes upon knockdown of CPSF73 and CFIm25 overlapped with gene expression changes during quiescence as well, but fewer genes were involved (Additional file 1: Figure S5C).

Some of the genes that were regulated (APA changes or gene expression changes) with knockdown of CstF-64 was found to be associated with GO terms related to cell movement (Table 3). Several of these migration genes that undergo changes in APA upon CstF64 knockdown also did so with quiescence, such as Arp2/3 complex protein ACTR2 and CDC42 and RAC1-binding protein IQGAP1.

\section{Cleavage and polyadenylation factor recognition sites are more prevalent in genes that undergo alternative isoform use with quiescence}

To further understand the importance of different cleavage and polyadenylation site factors in the alternative use of polyadenylation sites with quiescence, we 
Table 3 List of genes involved in motility that are altered with CstF64 knockdown

\begin{tabular}{ll}
\hline Type of change (analysis) & Genes $^{\text {a }}$ \\
\hline Gene expression (DESeq) & VIM, WDR1, ACTN1, ACTB, MAPK1, MYH9, BDKRB1, SEMA5A, FN1, IFT43, MYO1B, LMNA, HMGB2, GREM1, \\
& TPM4, DDIT4, IL8, TUBB4B, TUBB, PDCD6 \\
Alternative polyadenylation (Relative & MAP4, SP100, DYNC112, TBX5, OSBPL8, RBFOX2, BMPR1A, LAMA4, DOCK7, RAP2C, RHOT1, LAMC1, ACTR2, \\
usage of distal site) & IQGAP1, RHOT2, APP, LMO4, CERS2, GNA12, EGFR, WASF2, AMFR, SUN1, AMOTL1, STAT3, BSG, EXT1, COL18A1, \\
& NTF3, SIAH1, DPP4, EMP2 \\
\hline
\end{tabular}

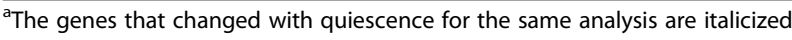

monitored the presence of their recognition motifs (Fig. 5a). For genes that undergo UR APA and shift to greater use of more distal polyadenylation sites during quiescence, their proximal polyadenylation site is more likely to have a strong hexamer (AAUAAA or AUUA AA), and less likely to have no hexamer, than for control genes (Fig. 5b). Similarly, when CPSF73 is knocked down, genes that shift to greater use of distal polyadenylation sites are less likely to have no hexamer than genes that do not lengthen with quiescence (Additional file 1: Figure S6). The findings support a role for reduced CPSF73 levels contributing to the use of more distal polyadenylation sites in genes undergoing UR APA in quiescent cells.

Extending the analysis to UGUA motifs recognized by CFIm25, among genes that use UR APA to shift to more distal polyadenylation site use in quiescent than proliferating cells, there was a significantly higher chance of a UGUA motif being present at the proximal site than for a control set of genes (Fig. 5c). With CFIm25 knockdown, the strongest effect was increased use of proximal polyadenylation sites, and the genes affected were more likely to have a UGUA motif at their distal polyadenylation site (Additional file 1: Figure S7).

To monitor the presence of binding sites for CstF-64, we determined the fraction of polyadenylation sites that contain a string of four or more uracils in the region 20-40 base pairs downstream of the polaydenylation site. With this analysis, there were more UUUU motifs at proximal polyadenylation sites among genes that shift to the use of more distal sites with quiescence, but the difference was not statistically significant (0.098) (Fig. 5d). We also monitored the fraction of U's (U-rich) and the fraction of U's or G's (UG-rich) in the same 20-40 base pair region. Proximal polyadenylation sites were enriched in U-rich and UG-rich sequences for genes that shifted to greater use of longer isoforms with quiescence (Fig. 5e and Additional file 1: Figure S8). This result is consistent with downregulation of CstF-64 playing a role in the shift to more distal polyadenylation sites with quiescence. Thus, in proliferating conditions, CstF-64 levels are more available for binding to U-rich proximal sites, which supports the generation of shorter isoforms.
Shifting to more distal polyadenylation sites stabilizes transcripts in quiescent but not proliferating fibroblasts Changes in the levels of transcripts that terminate at different polyadenylation sites could reflect changes in the rates that these isoforms are generated based on the levels of polyadenylation factors, or changes in the rates at which they decay. To understand the relationship between polyadenylation site selection and transcript fate, we first determined whether APA with quiescence was associated with a change in gene expression. Relative expression in quiescent compared with proliferating fibroblasts was slightly higher on average for genes that undergo a shift to greater use of distal polyadenylation sites with quiescence than for genes that do not undergo APA or use the proximal polyadenylation site preferentially in quiescence (Fig. 6a, $p<0.001$, Wilcoxon signed-rank test). This finding would be consistent with longer transcripts being more stable.

To better understand the relationship between polyadenylation site selection and transcript decay rate, we added actinomycin D to inhibit new transcription in proliferating or $7 \mathrm{dCI}$ fibroblasts, collected RNA over a timecourse, and performed polyadenylation site-enriched RNA-Seq to monitor the rate that different gene isoforms decayed [62]. The results extend our previous studies of genome-wide transcript decay rates in proliferating and $7 \mathrm{dCI}$ fibroblasts using microarrays [63]. In two different fibroblast strains (12-1 and 12-3), we found that isoforms terminating at distal polyadenylation sites were more stable than isoforms terminating at proximal polyadenylation sites in quiescent, but not proliferating, fibroblasts (Additional file 12 and Fig. 6b, c).

We identified motifs enriched in the interpolyadenylation site regions in genes that shift to a longer isoform with quiescence. Among the RNA-binding proteins that bind to these motifs, some are induced in quiescent compared with proliferating cells and would be candidates for stabilizing longer transcripts in quiescent cells (Additional file 1: Table S5). Our findings indicate that the shift to the use of longer isoforms in quiescent cells results in an overall stabilization of transcripts and a modest increase in expression levels. Therefore, the higher levels of longer isoforms in quiescent than proliferating fibroblasts could reflect both a difference in polyadenylation site selection (influenced by 


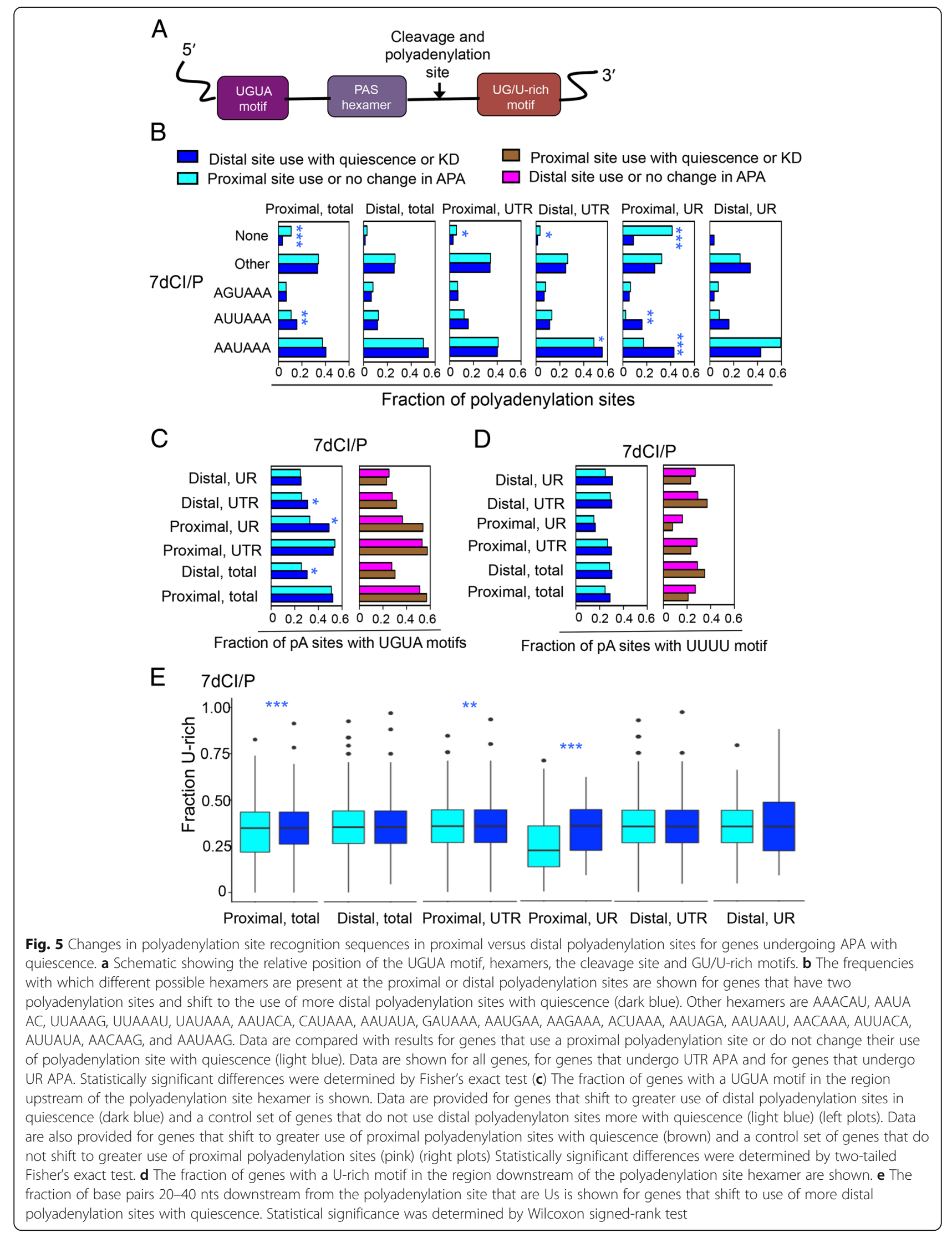



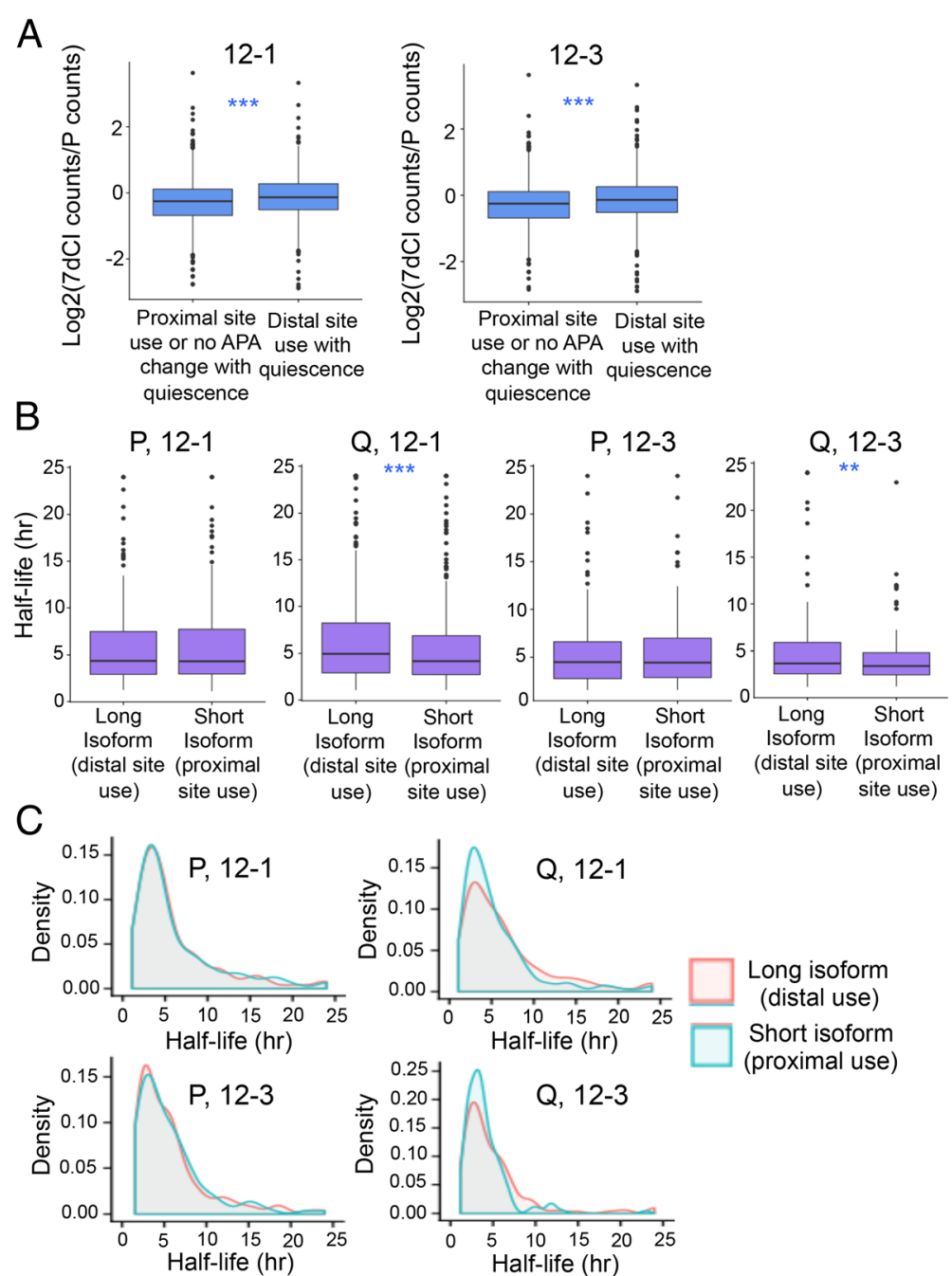

Fig. 6 Higher expression and increased stability for genes that shift to greater reliance on distal polyadenylation sites in quiescence. a For two different fibroblast lines (12-1 and 12-3), the $\log _{2}(7 \mathrm{dCl}$ counts/Proliferating counts) is plotted for genes that shift to increased use of more distal polyadenylation sites with quiescence and a control group that does not shift to more distal site use. Boxes indicate 25 to $75 \%$ ranges and whiskers indicate minimum and maximum values. Statistical significance was determined with Wilcoxon signed-rank test. The ratio of expression level in $7 \mathrm{dCl}$ versus $\mathrm{P}$ was higher for genes that shift to more distal polyadenylation site use with quiescence for both $12-1$ and $12-3$ fibroblasts. b Isoform-specific transcript decay half-lives were determined for 12-1 and 12-3 strains of fibroblasts in proliferating and quiescent conditions. Box plots show the range of half-lives for isoforms that terminate at proximal polyadenylation sites and isoforms that terminate at more distal polyadenylation sites in proliferating and quiescent conditions. Long isoforms are significantly more stable in quiescent but not proliferating states in 12-1 and 12-3 fibroblasts. Statistically significant differences were determined by Wilcoxon signed-rank test. c Density plots of half-lives for isoforms that terminate at proximal or distal polyadenylation sites in proliferating and quiescent fibroblasts from strains $12-1$ and $12-3$

levels of polyadenylation factors) and a difference in the rate at which the shorter and longer transcripts decay in the two proliferative states.

\section{Cleavage and polyadenylation factors are expressed at} higher levels in wound-healing than quiescent skin in vivo Wound healing is a situation in which cells are activated to both proliferate and migrate. We investigated the levels of cleavage and polyadenylation factors in normal skin and in dermal excisional wounds in mice. We introduced punch biopsies into the backs of mice and collected wounded tissue and unwounded control skin approximately $2 \mathrm{~cm}$ from the wound. Immunohistochemistry for the proliferation marker Ki-67 revealed higher levels of proliferation of a migrating mass of cells that includes fibroblasts, myofibroblasts, and immune cells in the skin proximal to the wound compared with cells in the dermis of control, unwounded skin (Fig. 7) [64]. Immunostaining for histone $\mathrm{H} 4$ as a control revealed similar staining in wounded and control skin as expected. Immunohistochemistry for CstF-64, CPSF73, or CFIm25 revealed a higher fraction of cells with 


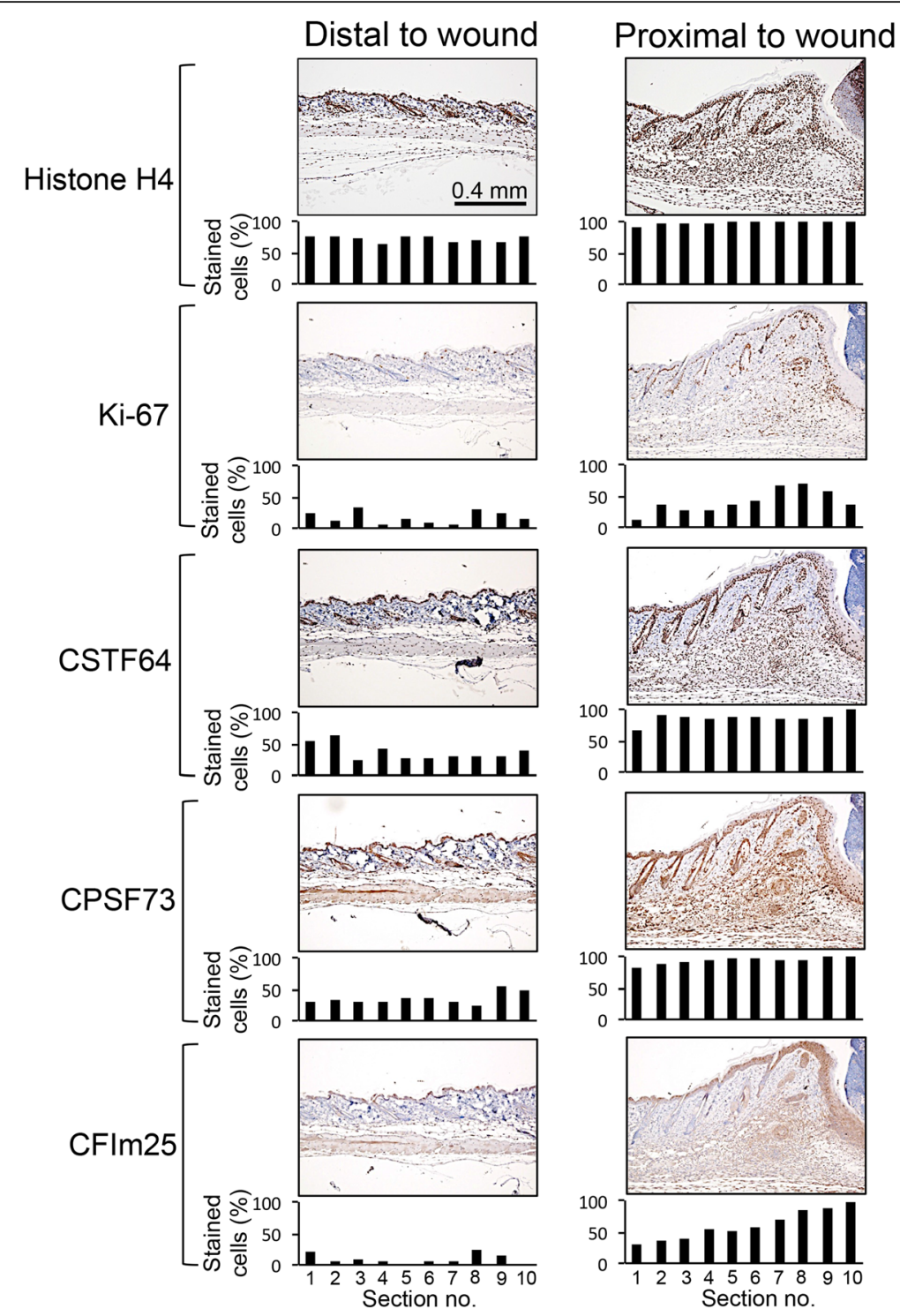

Fig. 7 Cleavage and polyadenylation factors are expressed at higher levels in fibroblasts near a wound than in fibroblasts of healthy skin. Mouse skin was collected 5 days after introduction of a punch biopsy. Normal mouse skin was collected $2 \mathrm{~cm}$ away from the wound. Samples were stained with immunohistochemistry for proliferation marker Ki-67, histone $\mathrm{H} 4$ as a control, or alternative polyadenylation and cleavage factors CstF-64, CPSF73 or CFIm25 (brown). Samples analyzed with immunohistochemistry were counterstained with hematoxylin (blue nuclei). Individual cells at different positions from the wounds were assigned positive or negative staining and the percentages are shown. Ki-67 does not label all dividing cells, and likely underestimates the fraction of cells that are actively cycling [122]. Levels of all three cleavage and polyadenylation factors were higher in the fibroblasts, myofibroblasts and immune cells proximal to a wound than in the fibroblast-rich dermal areas of healthy skin distal to the wound

positive nuclei in the region surrounding the wounded skin for all three factors than in control, unwounded skin (Fig. 7). This analysis revealed that the shift toward higher levels of cleavage and polyadenylation factors in proliferating fibroblasts in culture also occurs in the migratory, proliferating cells that heal wounds in vivo.

\section{CstF-64 knockdown reduces fibroblast migration}

Based on the consistency with which we observed changes in the mRNA processing and expression of genes important for cell motility in proliferating versus quiescent fibroblasts (Table 1), we hypothesized that changes in mRNA processing associated with the transition between proliferation and quiescence are also important for the closely linked process of cell migration. First we tested the association between proliferation and migration. We generated fibroblasts that were proliferating, induced into quiescence by $7 \mathrm{dSS}$, or restimulated after $7 \mathrm{dSS}$ by re-addition of medium with serum. We monitored the rate at which fibroblasts in each 
condition migrated into a denuded area on a tissue culture plate with real-time imaging (Fig. 8a). Migration was quantified as the ratio of cell concentration in the denuded area compared to the cell concentration in the non-denuded area, thus normalizing for possible differences in proliferation rate. We discovered that the proliferating and restimulated fibroblasts migrated into the denuded area more rapidly than the serum-starved fibroblasts (Fig. 8b).

We observed changes in the transcript and protein levels of cleavage and polyadenylation factors as fibroblasts transition between proliferation and quiescence. To test whether levels of cleavage and polyadenylation factors change in fibroblasts induced to migrate into a denuded area, we introduced denuded areas into cultures of fibroblasts and performed immunofluorescence to monitor the levels of cleavage and polyadenylation factors. CstF-64 and CPSF73 levels were significantly higher in the cells that had migrated into the denuded area than cells that had not migrated, while no significant change was observed for CFIm25 (Additional file 1: Figure S9). We then tested the importance of alternative polyadenylation factors for fibroblast motility. We generated knockdown fibroblasts with control siRNAs or siRNAs against cleavage and polyadenylation factors, and monitored the rate of migration. Knockdown of CstF-64 with any of three different siRNAs (Fig. 8c) resulted in reduced migration into the denuded area (Fig. 8d). CstF-64 siRNA \#1 had the strongest effect on CstF-64 levels and resulted in the most significant reduction in migration. Knockdown of CPSF73 (Fig. 8c) resulted in slower migration, but the difference was not statistically significant (Fig. 8d). Knockdown of CFIm25 (Fig. 8c) did not affect migration rate (Fig. 8d). Thus, CstF-64 is induced in migrating cells, and knockdown of CstF-64 resulted in APA changes and downregulation of genes that overlap with those that occur with quiescence, including genes associated with cell migration (Table 3). These findings are consistent with our observation here that knockdown of CstF-64 simulates the reduced migration observed for quiescent fibroblasts.

\section{Knockdown of cleavage and polyadenylation factors reduces migration of triple negative breast cancer cells}

To determine the generality of our findings for different types of cells, we tested the effects of siRNAs targeting CstF-64, CPSF73 or CFIm25 on the migration of triple negative breast cancer cells (Additional file 1: Figure S3). Triple negative breast cancer is a highly aggressive breast cancer subtype characterized by a lack of hormonal receptors and an absence of HER2 amplification [65]. Knockdown of CstF-64 or CPSF73 resulted in significantly reduced migration of triple negative breast cancer cells (Fig. 8e). The triple negative breast cancer cells were even more sensitive to altered polyadenylation site selection than the fibroblasts, which may reflect the increased reliance of cancer cells on proximal polyadenylation sites $[20,45,46,66]$. Our results demonstrate that the selection of polyadenylation sites can affect the migratory capacity of cancer cells as well as fibroblasts in wound healing (Fig. 8f).

\section{Discussion}

While we and others have shown that the transition to quiescence is associated with widespread changes in gene expression [9-11], and others have previously shown changes in the selection of polyadenylation sites with quiescence [21], we sought here to better understand the relationship between quiescence and alternative polyadenylation. Gene expression analysis of RNA-Seq data revealed that genes involved in mRNA processing (splicing and polyadenylation) are downregulated as fibroblasts enter quiescence (Fig. 1c, d). These findings suggested to us that processing of pre-mRNA transcripts may be different in quiescent compared with proliferating cells, and that these changes may contribute to changes in transcript abundance and the functional attributes of proliferating versus quiescent fibroblasts. We further discovered through differential exon analysis of RNA-Seq data that hundreds of genes exhibit changes in isoform expression during the transition to quiescence. Quiescent fibroblasts expressed lower levels of some auxiliary splicing factors (Fig. 2b) and were more likely to include exons and retain introns than proliferating fibroblasts (Fig. 2a), demonstrating cell-cycle state-dependent changes in splicing and intron retention [38]. Introns that were retained tended to have splicing motifs that varied from the consensus sequence, especially for the polypyrimidine tract adjacent to 3' splice sites in the proliferating state (Fig. 2c), potentially reducing the effectiveness of splicing factors or associated RNA binding proteins. Our results are consistent with a model in which quiescence is associated not with a complete shut-down of mRNA processing events, but rather with a shift in the processing of specific transcripts such that, in addition to changes in gene expression, an alternative set of exons and isoforms are present in fibroblasts that are proliferating versus quiescent. Genes involved in cell motility were among those demonstrating consistent changes in splicing in proliferating versus quiescent cells (Table 1).

Among the changes in isoform use that we observed, the most prominent effect was a change in the selection of polyadenylation sites in proliferating versus quiescent fibroblasts. In response to quiescence induced by contact inhibition, 714 genes exhibited a change in polyadenylation site selection, and in $88 \%$ of instances, alternative polyadenylation site use resulted in a lengthening of 
A

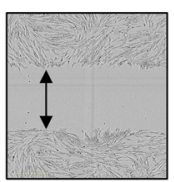

$\mathrm{Ohr}$

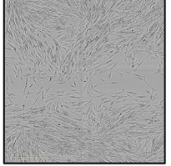

$12 \mathrm{hr}$

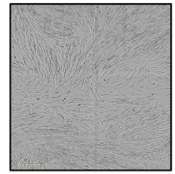

$24 \mathrm{hr}$
B

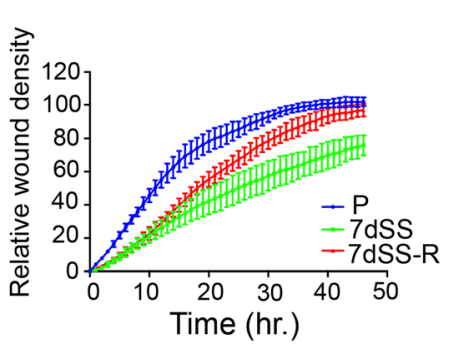

SiRNA
C

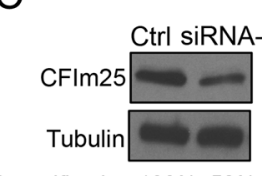

Quantification 100\% 52\%

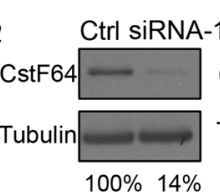

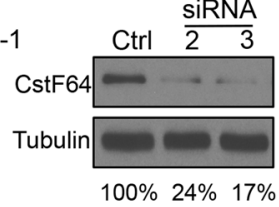

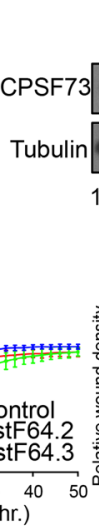

Ctrl $\frac{\text { siRNA }}{12}$
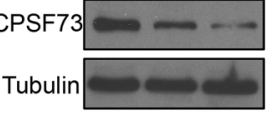

$100 \% \quad 54 \% \quad 28 \%$
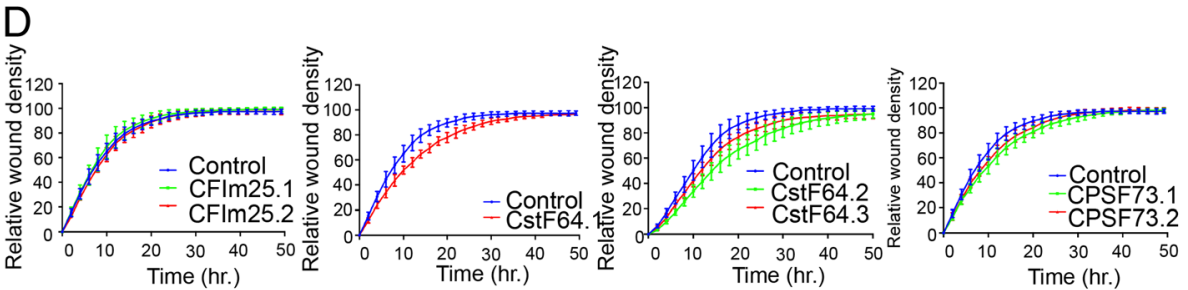

$E$
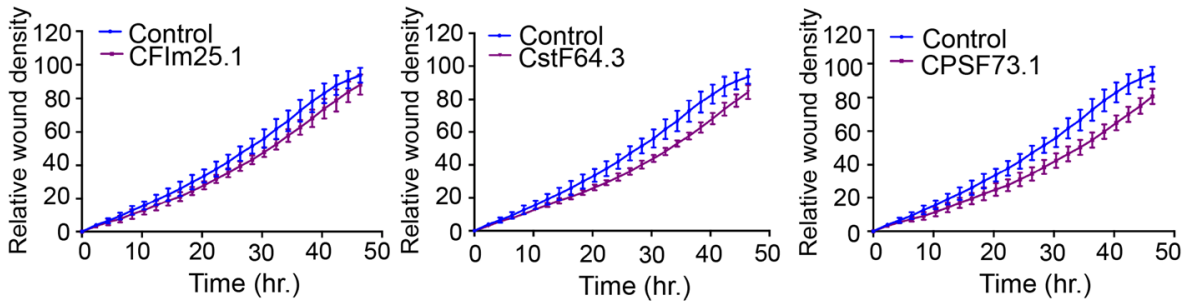

F

\section{Unwounded tissue}

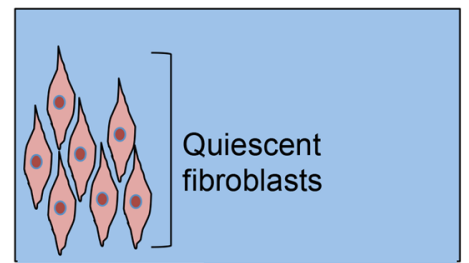

Wounded tissue

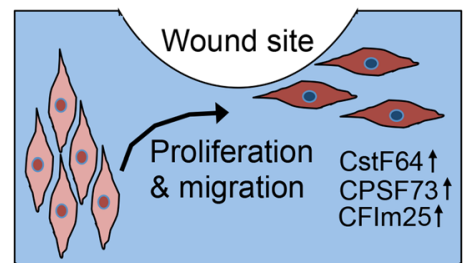

Fig. 8 Knockdown of APA factors results in reduced migration. a Example of Incucyte migration assay. Bright-field images from an assay monitoring the rate of migration into a denuded area (marked by a double-arrow) performed with Incucyte real-time imaging are shown. $\mathbf{b}$ Proliferating fibroblasts migrate more rapidly into a denuded area than quiescent fibroblasts. Fibroblasts were sampled in proliferating conditions, 7dSS conditions (7dSS), or after 7dSS followed by serum restimulation (7dSS-R). Fibroblasts were plated into 96-well plates and a portion of the well was denuded of cells. Plates were analyzed with an Incucyte real-time imaging instrument and the associated software to monitor the rate at which fibroblasts migrated into the denuded area. The ratio of cell density in the denunded area to the non-denuded area (relative wound density) over a time-course is plotted. Six wells were monitored for each condition and data represent mean and standard deviation. Proliferating versus 7dSS samples ( $p$ value $<0.001$, repeated measures two-way ANOVA with Dunnett's multiple comparison test), proliferating versus 7dSS-restimulated samples ( $p$ value $<0.001$ ), and 7dSS versus 7dSS-R ( $p$ value $<0.001$ ) were statistically significantly different. $\mathbf{c}$ Immunoblots demonstrating knockdown of the targeted cleavage and polyadenylation factor by siRNAs in fibroblasts. The percent knockdown of protein level is also shown. d Knockdown of CstF-64 reduces fibroblast migration. Fibroblasts were transfected with a control siRNA or an siRNA against CFIm25, CstF-64, or CPSF73. CstF-64-knockdown fibroblasts exhibited reduced migration into a denuded area than control fibroblasts (CstF64.1 $p$ value $=0.0013)$. Two additional siRNAs against CstF-64 (CStF64.2 and CstF64.3) reduced migration compared with a matched control siRNA as well (CstF64.2 p value $=0.0021$, CstF$64.3 p$ value $=0.0384$ ). Six replicates were performed for each condition. e Knockdown of CStF-64 or CPSF73 reduced migration of triple negative breast cancer cells. Triple negative breast cancer cell line MDA-MB-231 was transfected with a control siRNA or an siRNA against CstF-64, CPSF73 or CFIm25. Migration into a denuded area on the plate was monitored with an Incucyte instrument. Knockdown of CstF-64 or CPSF73 resulted in reduced migration (CStF64 $p$ value $=$ 0.0002 , CPSF73 $p$ value $=0.0013$ ). For all conditions, the number of replicates for each condition was $6 . \mathbf{f}$ Schematic diagram showing elevated cleavage and polyadenylation factors in fibroblasts in the wound-healing environment. Increased expression of CstF-64, CPSF73, and CFIm25 in fibroblasts in wounds is expected to result in increased use of proximal polyadenylation sites and may promote fibroblast migration to the wound 
transcripts in quiescent compared with proliferating cells (Fig. 3c). These findings are consistent with previous studies that revealed that $3^{\prime}$ UTRs are shorter in more rapidly proliferating cells $[18,21]$, stem cells [67], and cells and tissues derived from tumors [20, 46, 68], and longer in cells that divide less frequently such as differentiated tissues [13, 15, 67]. We found that 3' UTR lengthening reverses when quiescent cells re-enter the cell cycle (Fig. 3b), demonstrating that these changes can be reversed based on proliferative state.

To better understand the basis for the changes in polyadenylation site selection in proliferating versus quiescent fibroblasts, we monitored the levels of polyadenylation factors in proliferating and quiescent cells. Transition to quiescence was associated with lower levels of cleavage and polyadenylation factors CstF-64, CFIm25, and CPSF73 (Fig. 3d). Knockdown of each these three factors resulted in changes in polyadenylation site use that overlapped significantly with the changes that occurred with quiescence (Fig. 4b and Additional file 1: Figure S5A and $\mathrm{B})$. There were also changes in gene expression as a result of knockdown of specific factors, especially CstF-64. These gene expression changes overlapped with changes in gene expression that occur with quiescence (Fig. 4c and Additional file 1: Figure S5C).

To further understand the contribution of different cleavage and polyadenylation complexes to the shift in polyadenylation site selection with quiescence, we monitored the presence of their recognition sites. For genes that use more distal upstream region polyadenylation sites with quiescence, the proximal hexamer was much more likely to match the canonical hexamer, and very unlikely to be absent (Fig. 5b). A similar shift was observed with CPSF73 knockdown (Additional file 1: Figure $\mathrm{S6A}$ ). This is consistent with reduced expression of CPSF73, and reduced use of upstream region proximal polyadenylation sites, as a factor contributing to the lengthening of transcripts with quiescence. A role for reduced CstF-64 levels in quiescent cells promoting the shift to more distal polyadenylation sites is supported by the finding that the sequence between 20 and 40 bps downstream of the proximal polyadenylation site included more Us on average and more Gs and Us on average, for genes that use more distal polyadenylation sites with quiescence (Fig. 5e). Taken together, the results support the importance of reduced levels of cleavage and polyadenylation factors with quiescence, with the polyadenylation pattern for specific sequences determined in part by the presence or absence of binding factors for the reduced factors.

Some previous studies have reported that shorter transcripts generated by alternative polyadenylation tend to be expressed at higher levels than the corresponding longer isoform [20,46, 69, 70], while other studies have found little effect of alternative polyadenylation on transcript levels, transcript stability or protein abundance $[71,72]$. Additional studies have found that shorter transcripts can be more or less stable [71, 73], and two detailed analyses in yeast showed clear examples of stability elements in 3' UTRs that make longer isoforms more stable than shorter isoforms [74, 75]. In our study, we observed that genes with longer 3' UTRs during quiescence, on average, exhibited a small but significant increase in expression level during quiescence compared to proliferating cells (Fig. 6a). Further, isoforms are more stable when distal rather than proximal polyadenylation sites are used in the quiescent state, but decay rates are similar when proximal or distal sites are used in the proliferating state (Fig. 6b, c). The findings are consistent with induction of an RNA-binding proteins in quiescent cells that bind to motifs present in the region between the polyadenylation sites and limit transcript degradation when the cells are quiescent. There are multiple motifs recognized by RNA-binding proteins in this inter-polyadenylation site region, and some of the factors that recognize these motifs are expressed at higher levels in quiescent than proliferating fibroblasts (Additional file 3). The findings are also consistent with the retention of longer transcripts in ribonucleoprotein storage granules or other structures in quiescent cells [76]. These changes could contribute to the higher gene expression levels of transcripts undergoing transcript lengthening in quiescence (Fig. 6a).

In many [20], but not all [77], studies, cancerous tissue and cancer cell lines were found to be more likely to express transcripts that terminate at proximal than distal polyadenylation sites, consistent with our observations in proliferating fibroblasts. Different polyadenylation factors have been found to have distinct effects on APA. Downregulation of CFIm25 repressed proximal polyadenylation site use (Additional file 1: Figure S4C) consistent with previous reports [45, 54]. Depletion of CFIm25 has been found to enhance the tumorigenic properties of glioblastoma cells [45], while overexpression of CFIm 25 reduced tumor growth [45]. Shortening of 3' UTRs has been associated with poor prognosis in breast and lung cancer [78]. Further, in an analysis of multiple tumor datasets deposited in The Cancer Genome Atlas, expression of CstF-64 correlated most closely with shortening of transcripts, with CPSF73 showing the next best correlation among the factors investigated [46]. Expression of shorter 3' UTRs was an important predictor of patient outcome even beyond established clinical attributes [46]. In another study, CstF-64 expression was found to be associated with poor prognosis in lung cancer and its overexpression increased lung cancer cell proliferation and invasion [79]. In our dataset, cyclin D1 was the most strongly downregulated gene when CstF-64 was knocked down (Additional file 11), 
raising the possibility that CstF-64 levels modulate polyadenylation site selection and cyclin levels. Taken together with our data demonstrating that downregulation of CstF-64 in triple negative breast cancer cells reduces their migration (Fig. 8e), the data as a whole suggest that CstF-64-mediated APA may play an important role in regulating polyadenylation site selection, gene expression, cancer cell migration, metastasis, and patient outcome.

Fibroblasts transition from quiescence to proliferation and become more migratory in the context of wound healing. Some previous studies have supported a role for mRNA processing in wound healing [80-83]. By investigating the wound healing response in mice, we found that the levels of polyadenylation factors CstF-64, CFIm25, and CPSF73 were significantly higher in the area adjacent to the wound than distal to the wound (Fig. 7), similar to our finding that these factors are expressed at higher levels in proliferating than quiescent fibroblasts in culture (Fig. 3d). The results support a possible role for alternative polyadenylation in the proliferative and migratory changes that occur in the wound healing process.

Previous studies have identified mechanistic links between fibroblast proliferation and migration. Mitogen binding to receptor tyrosine kinases can activate focal adhesion kinase (FAK) and thereby stabilize focal adhesions $[84,85]$. Activation of receptor tyrosine kinases can also recruit WASp [86], which promotes the formation of branched actin filaments that promote cell migration. The anti-proliferative cyclin-dependent kinase inhibitor p2 $7^{\text {Kip1 }}$ binds to and inhibits the activity of RhoA GTPase [87], an important regulator of actin dynamics and adhesion, spreading and migration [88]. Our findings that downregulation of APA factors, as occurs in response to antiproliferative signals via E2F transcription factors [21], reduces the capacity of fibroblasts to migrate into a denuded area, represents another mechanism linking fibroblast proliferation to migration through APA. We found that CstF-64 is induced in migrating cells, and knockdown of CstF-64 resulted in changes in polyadenylation site selection, altered expression of several migration genes (Table 3), and reduced cell migration (Fig. 8d). Among the genes expressed at lower levels with CstF-64 knockdown are beta actin, $\alpha$-actinin, and myosin $1 \mathrm{~b}$. Our findings support a model in which changes in the selection of polyadenylation sites or changes in gene expression mediated by the levels of alternative polyadenylation factors play an important role in critical cell functions including migration. In a separate manuscript, we investigate in more detail the effects of isoform changes in one particular gene, RECK (included in Table 1 under UR-APA), on migration [89]. Taken together, our data and the data emerging from other laboratories, underscore the importance of CstF-64 as an important regulator of cellular functions, including migration, in multiple cellular contexts.

\section{Conclusions}

Our work demonstrates that, in addition to changes in gene expression, the shift from a proliferating to a quiescent state is associated with changes in intron and exon inclusion and with the selection of polyadenylation sites. Overall, quiescent cells tend to retain introns and express longer transcripts that are present at higher levels and are more stable. Cleavage and polyadenylation factor CstF-64 is more abundant in proliferating fibroblasts in culture and in fibroblasts near a denuded area or a wound in mice. Knockdown of CstF-64 recapitulates changes in isoform use and gene expression in quiescent cells, and results in reduced cell migration in fibroblasts and cancer cells. Fibroblasts are often induced to proliferate and migrate in similar situations, and our data indicate that changes in the levels of CstF-64 can serve as a link between proliferative cues and migratory capacity.

\section{Methods \\ Cell culture}

Human foreskin fibroblasts were isolated from human skin obtained from the National Disease Research Interchange (NDRI) as described previously [24, 90]. Cells were seeded at $5 \times 10^{5}$ cells per $10 \mathrm{~cm}$ dish for each cell cycle state and grown in Dulbecco's modified Eagle medium (DMEM) (Life Technologies, Grand Island, NY) supplemented with $10 \%$ fetal bovine serum (FBS) (Atlanta Biologicals, Flowery Branch, GA and Corning, Thermo Fisher Scientific, Waltham, MA) at $37^{\circ} \mathrm{C}$ in a $5 \% \mathrm{CO}_{2}$ incubator. Detailed procedures for culturing proliferating and quiescent fibroblasts are described in [91]. Briefly, proliferating fibroblasts were collected for analysis 2 days after plating (60-80\% confluent). $7 \mathrm{dCI}$ fibroblasts were collected 7 days after plating, or at an equivalent density, while $7 \mathrm{dSS}$ fibroblasts were seeded in full serum medium (10\% FBS in DMEM), changed to reduced serum medium (0.1\% FBS in DMEM), and collected 7 days after adding the reduced serum medium. Medium was changed every 2 days for both $7 \mathrm{dCI}$ and $7 \mathrm{dSS}$ fibroblasts. Restimulated samples were prepared by first performing the relevant quiescence arrest and readding the limiting factor. Restimulated fibroblasts were monitored with Incucyte migration assays or collected 24 or $48 \mathrm{~h}$ later for real-time PCR analysis. The triple negative breast cancer cell line MDA-MB-231 cell line (generous gift of the Banerjee and Christofk laboratories) was grown in $10 \%$ FBS in DMEM.

\section{RNA isolation for RNA-Seq and microarray analysis}

RNA-Seq was performed on three biological replicates of fibroblasts isolated from two different donors, 12-1 and 10-5. Medium was aspirated from tissue culture plates of fibroblasts, and the attached cells were washed with $5 \mathrm{ml}$ of PBS. Attached fibroblasts were lysed into $1 \mathrm{~mL}$ of Trizol reagent (Life Technologies, Carlsbad, 
CA) per $10 \mathrm{~cm}$ plate for $5 \mathrm{~min}$. RNA was isolated from Trizol lysates as previously described [92, 93]. RNA concentrations were determined using a Nanodrop Spectrophotometer (Thermo Fisher Scientific Inc., Waltham, MA). RNA quality was verified on a Bioanalyzer 2100 (Agilent Technologies, Santa Clara, CA) using reagents from the RNA Nano 6000 kit (Agilent Technologies).

\section{RNA-Seq analysis}

cDNA libraries were constructed using the Illumina TruSeq mRNA sample preparation kit (Illumina Inc., San Diego, CA) according to the manufacturer's instructions for revision A of the protocol (Illumina Part \#15008136). The low-input protocol was followed for all samples, and 1 to $10 \mu \mathrm{g}$ of total RNA input was used per library (unstranded). Single-end $140 \mathrm{bp}$ reads were generated on an Illumina HiSeq 2000 Instrument. Reads with Illumina (PHRED-based) quality scores above 10 (90\% accuracy) were mapped to the hg19/GRCh37 build of the human genome using the TopHat (version 2.0.9) genome alignment algorithm [94, 95]. The bowtie indices for human were obtained from the bowtie website: http://bowtie-bio.sourceforge.net/tutorial.shtml. The standard workflow for Tophat alignment was followed as described here: https:// ccb.jhu.edu/software/tophat/manual.shtml.

The default parameters for alignment as described in the Tophat manual were used. Standard DESeq (version 1.22.0) workflow [96] (https://bioconductor.org/packages/ release/bioc/html/DESeq.html) was used to convert the output of TopHat (BAM files) to a file format with gene identifiers (UCSC gene annotation, GRCh37/hg19 assembly, date of access June, 2013) and read counts normalized for sequencing depth across the different biological samples and cell cycle conditions. Information about biological replicates was provided as input for variance calculations to determine differential expression among proliferating and $7 \mathrm{dCI}$ conditions in DESeq. To identify differentially expressed genes, the $\log _{2}$ (7dCI read count/ proliferating read count) was used to compare expression differences between the two states. Genes with differences in read counts between conditions (proliferation versus $7 \mathrm{dCI}$ ), and low variance in expression within the three biological replicates of each condition, were called significant by DESeq after multiple hypothesis correction (FDR $<5 \%$ ) [97]. Heat maps were generated using the heatmap2 function of gplots package (2.12.1) (https://cran.r-project.org/web/packages/gplots/index.html) implemented in the $\mathrm{R}$ programming language $[98,99]$.

\section{Gene set enrichment analysis}

For RNA-Seq data, gene sets with significantly different expression between proliferating and quiescent fibroblasts were identified using a Wilcoxon rank-sum test comparing the log fold-change estimates of genes within each set to genes not within the set [100]. Graphics were created using the GSEMA package implemented in $\mathrm{R}$ [101].

\section{Differential isoform analysis}

To determine differential isoform use between proliferating and quiescent fibroblasts, the standard DEXSeq (version 1.14.2) workflow (https://bioconductor.org/ packages/release/bioc/html/DEXSeq.html) [29] was followed. BAM files generated by aligning RNA-Seq reads to the human genome (hg19/GRCh37 build) were converted to gene-normalized read count files using exons as the identifiers. The Ensembl gene annotation (GRCh37 assembly) file was obtained from https:// ccb.jhu.edu/software/tophat/igenomes.shtml. Differential exon expression was determined across the three biological replicates. Genes with significant differences in expression for specific exons (adjusted $p$ value $<0.05$ ) between proliferating and $7 \mathrm{dCI}$ conditions were used for further analysis.

\section{Microarray gene expression analysis}

An aliquot of the same total RNA that was analyzed by RNA-Seq was also analyzed by microarray. Total RNA was reverse-transcribed into cDNA and fluorescently labeled with Cyanine 3-CTP (7dCI samples) or Cyanine 5-CTP (proliferating samples) with the Quick Amp Labeling Kit for Microarray Analysis (Agilent Technologies, Santa Clara, CA) following the manufacturer's protocol. cRNA samples that passed yield and labeling standards were fragmented, and proliferating and quiescent samples were hybridized to two-color Human gene expression $4 \times$ $44 \mathrm{~K}$ microarrays (Agilent Technologies) for $17 \mathrm{~h}$ at $65^{\circ} \mathrm{C}$ in an oven rotating the arrays at 10 rotations per minute. Fluorescence intensities were detected using the Genepix scanner (Agilent Technologies) and probe identities were determined using Agilent's feature extractor version 11.5. Probes detected over background fluorescence thresholds were used in subsequent gene expression analyses to calculate $\log _{2}\left(7 \mathrm{dCI}_{\text {intensity }} / \mathrm{P}_{\text {intensity }}\right)$.

\section{Differential splicing analysis}

RNA-Seq reads (fastq files) from three replicates of proliferating fibroblasts and three replicates of $7 \mathrm{dCI}$ fibroblasts were analyzed with the rMATS algorithm release 3.2.1 (http://rnaseq-mats.sourceforge.net/rmats 3.2.1.beta/) [31-33] using Ensembl gene annotation (GRCh37 assembly). Reads were trimmed to a length of $100 \mathrm{bps}$ for analysis using the Trim Fastq tool provided as part of rMATS package. Standard workflow for rMATS (default parameters as described in: http://rnaseq-mats.sourceforge.net/rmats3.2.1.beta/user_guide.htm) was used 
for the splicing analysis using the reads that cover the splicing junctions and target regions. Alternative splicing events with an FDR of $<0.05$ were considered statistically significant.

\section{Polyadenylation site-enriched RNA-Seq}

We performed polyadenylation site-enriched RNA-Seq with two methodologies (Gnomegen [89] and Nextera). Here we describe the second approach, Nextera. For polyadenylation site-enriched RNA-Seq, two different primary dermal fibroblasts, $12-1$ and $12-3$, were used as biological replicates. Proliferating, 7dCI, and siRNAtreated fibroblasts were lysed by adding $1 \mathrm{ml}$ of Trizol per $10 \mathrm{~cm}$ plate and incubating the plate for $5 \mathrm{~min}$ at room temperature. RNA was isolated from the cell lysates using the Direct-zol ${ }^{\mathrm{Tm}}$ RNA MiniPrep Plus kit (Zymo Research, Irvine CA) by following the manufacturer's instructions. The concentration of RNA was measured using Nanodrop 2000c (Thermo Fisher Scientific). cDNA libraries containing fragments enriched for 3'UTR ends were created with the Nextera kit using the Smart-seq2 cDNA amplification method as described in [102]. Common forward primers were used for all samples; reverse primers with a unique barcode sequence (i5 indices) were specific for each sample. The size distribution of the cDNA library was confirmed using a High Sensitivity DNA chip (Agilent Technologies) on a Bioanalyzer 2100 Instrument (Agilent Technologies). Libraries with a uniform size distribution between 150 and $1000 \mathrm{bp}$ were subjected to gel size selection to enrich for 180-280 bp sized fragments. The concentration of the final library was measured on a qubit fluorometer (Thermo Fisher Scientific). Single-end 150 bp reads were generated on an Illumina HiSeq 2500 Instrument. The sequencing reaction was run for 150 cycles.

\section{Polyadenylation site-enriched RNA-Seq analysis}

Reads from polyadenylation site-enriched cDNA libraries were demultiplexed followed by removal of adapter and polyA tail sequences. Trimmed reads were aligned to the human genome (hg19/GRCh37 build) using TopHat (version 2.0.14) [94] using default parameters. Aligned reads were assigned to a polyadenylation site based on annotations in the Poly(A)site atlas (version:r1.0(hg19) by Gruber et al. [103] using the Perl script provided (http://www.polyasite.unibas.ch/). Only the polyadenylation sites annotated as TE (terminal exon), EX (any other exon except the terminal one), or IN (any intron), and with at least 10 counts across all the samples, were included for analysis. For genes containing two polyadenylation sites, the relative use of the distal polyadenylation site (RUD) [13, 18] was determined as distal polyadenylation counts/total read counts (distal plus proximal counts). The RUD values for two biological replicates were averaged to determine the RUD value of a gene. Changes in alternative polyadenylation between the two conditions were significant if the RUD difference between them was greater than 0.05. For genes with more than two polyadenylation sites, a parameter called relative site usage (counts for a polyadenylation site divided by total counts for all the polyadenylation sites) was calculated for all the polyadenylation sites of a gene. To perform differential expression analysis, counts from all the polyadenylation sites of a gene were combined and the combined counts for all the genes for two different conditions were subjected to DESeq2 (version 1.18) analysis [96, 104] using standard parameters (Ensembl annotation, GRCh37 assembly).

\section{Transcript decay rate measurements}

Detailed protocols for cell culture and actinomycin D treatment are described in $[63,105]$. Briefly, to monitor transcript decay rates, proliferating and $7 \mathrm{dCI}$ fibroblasts were treated with $15 \mu \mathrm{g} / \mathrm{ml}$ actinomycin D (Sigma-Aldrich, Inc., St. Louis, MO). Cells were washed with PBS and cell lysates were collected using Trizol reagent (Life Technologies) at 0, 120, 240, and $480 \mathrm{~min}$ after addition of actinomycin D. RNA was isolated from Trizol lysates using the Direct-zol ${ }^{\mathrm{TM}}$ RNA MiniPrep Plus kit (Zymo Research). cDNA library preparation, sequencing, and processing of reads were performed as described for polyadenylation-site enriched RNA-Seq.

\section{Decay rate calculations}

For comparisons of decay rates under different conditions, only the genes with two polyadenylation sites (proximal and distal) in the 3' UTR were used for analysis. Further, only transcripts with a minimum of 10 counts at $t=0$ were used. For each polyadenylation site, the counts at four time points $(0,2,4$, and $8 \mathrm{~h})$ were log-transformed and fit to a linear decay model ([63, 105]) using the least squares method to determine a fitting parameter $\left(R^{2}\right)$ and to obtain decay constants. Only the polyadenylation sites with $R^{2}$ value greater than 0.6 were used. The decay constants $(k)$ were converted to half-lives $(\ln 2 / k)$ for isoform-specific analysis.

\section{Motif analysis}

For all of the transcripts that undergo APA with quiescence and had two detectable polyadenylation sites, sequences (in FASTA format) were obtained from the UCSC Genome Browser (Table browser tool, hg19/ GRCh37 build, accessed on March 2018) that include the polyadenylation site itself, 100 nts upstream (for UGUA motif analysis), and the region 20 to $40 \mathrm{nt}$ downstream (for U-rich and UG-rich motif analysis) of the polyadenylation site. For hexamer analysis, the hexamer associated with each of the polyadenylation sites was 
obtained from Poly(A)site atlas annotations (Homo sapiens-version:r1.0(hg19)) by Gruber et al. (http:// www.polyasite.unibas.ch/) [103]. For sites associated with more than one hexamer, we chose the hexamer with the highest signal strength as determined by Gruber et al. For UGUA analysis, FIMO (v4.12.0) [106] motif analysis tool of the MEME suite was used with $p$ value set to 1 to return matches to all of the UGUA motifs. Post-processing of the FIMO results was used to check for exact matches. For RBP motif analysis, primary sequences (in FASTA format) from the alternate region (region between proximal and distal sites in the 3' UTR) for genes that become longer (distal polyadenylation site use) with quiescence were extracted using the Table browser tool of the UCSC Genome Browser (hg19/GRCh37 build, accessed on March 2018). To generate a background dataset, all the sequences from alternate regions of genes that use more proximal sites with quiescence and genes with no change in polyadenylation site use with quiescence were used. RBP motifs enriched in primary sequences in comparison with background sequences were obtained using the analysis of motif enrichment (AME, v4.12.0) motif enrichment tool [107] of the MEME suite. The RNA motifs from Ray2013 Homo sapiens motif database [108] were used for enrichment testing. Only the RBP motifs enriched in both 12-1 and 12-3 biological replicates were considered. For U-rich and UG-rich analysis, the sequences of the regions encompassing 20 to $40 \mathrm{nt}$ downstream of the polyadenylation site for each gene were extracted for all genes with two polyadenylation sites using the Table browser tool of the UCSC genome browser (hg19/GRCh37 build, accessed on March 2018). The U-rich sequences in this region have been shown to be the preferred binding sites of CstF64 using crosslinking immunoprecipitation (CLIP)-Seq analysis [109]. Percent U was calculated by determining the fraction of Us present in this region. Percent UG was calculated by determining the sum of the fractions of Us and Gs present in this region. For analysis of 4-mer UUUU sequence [110], the presence or absence of a UUUU motif was determined in this region.

\section{Splicing site analysis}

Nucleotide sequences were extracted for the $5^{\prime}$ and 3' splice sites for 139,180 constitutive exons from HEXEvent online database [111] and for the introns called differentially retained (FDR <0.05) by rMATS in proliferating or quiescent fibroblasts (Additional file 4). For analyzing $5^{\prime}$ and $3^{\prime}$ splice sites, motifs of 9 bases (3 bases in the exon and 6 bases in the intron) and 23 bases (20 bases in the intron and 3 bases in the exon), respectively, were used. A position weight matrix was generated from constitutive exon $5^{\prime}$ and $3^{\prime}$ sequences using scripts written in the $\mathrm{R}$ programming language [112, 113].
Based on this position weight matrix, the probability of each sequence was determined for each sequence in the list of constitutive exons, introns retained in proliferating conditions and introns retained in quiescent conditions. Statistical significances of the groups of probabilities were determined with ANOVA with Tukey's multiple comparison test. Sequence logos were generated from the position weight matrix using the $R$ programming language (seqLogo package, https://bioconductor.org/packages/release/bioc/html/seqLogo.html) [114].

\section{Antibodies for immunoblotting}

Antibodies against tubulin (T6074) and CFIm25 (AV40695-100UG, 1:800 dilution) were obtained from Sigma-Aldrich, Inc. (Saint Louis, MO). An antibody against CstF-64 (sc-28201, 1:200) was purchased from Santa Cruz Biotechnology, Inc. (Dallas, TX). An antibody against U1-70K (06-1297, 1:2000) was purchased from EMD Millipore (Billerica, MA). Antibodies against CPSF73 (A301-090A-T), U2AF65 (A303-665A-T), FUS (A300-292A-T), and RNA Polymerase II Phospho S5 (A304-208A-T) were purchased from Bethyl Laboratories (Montgomery, TX) and used at 1:1000 dilution.

\section{Immunoblotting}

Immunoblotting was performed using a standard protocol similar to that described previously [7]. Briefly, cells were lysed using mammalian protein extraction reagent (MPER) (Thermo Fisher Scientific Inc., Waltham, MA) containing protease and phosphatase inhibitors (Roche Applied Science, Indianapolis, IN) according to the manufacturer's instructions (Thermo Fisher Scientific Inc.). Total protein concentrations in collected lysates were measured using Pierce ${ }^{\mathrm{Ta}}$ BCA protein assay kit (Thermo Fisher Scientific Inc.). Samples were run on SDS PAGE gels and transferred to polyvinylidene difluoride Immobilon-P membranes (EMD Millipore, Billerica, MA). Membranes were blocked with 5\% BSA in phosphate-buffered saline-Tween. Immunodetection was performed using primary and HRP-conjugated secondary antibodies based on standard protocols.

\section{Mouse wounding assays}

All experiments were approved by the UCLA Office for Animal Research, protocol number 2015-033. C57/BL6 mice were provided housing and husbandry in accordance with Institutional Animal Care and Use Committee approved protocols. Mice that were approximately 810 weeks of age were anesthetized, shaved, and provided with analgesia. We introduced one full thickness dermal punch biopsy of $3.5 \mathrm{~mm}$ on each mouse's upper back. On day 5 after wounding, the mouse was $83.6 \%$ healed. Mice were euthanized with $\mathrm{CO}_{2}$ followed by cervical 
dislocation. We excised the wound bed en bloc with the surrounding soft tissue and at least $0.5 \mathrm{~cm}$ of normal tissue surrounding the incision. We also collected normal skin from the same mice for comparison. Skin and wounds were fixed in formalin and paraffin-embedded. Slides were cut from paraffin blocks for immunohistochemistry.

\section{Immunohistochemistry}

Tissue slices $(4 \mu \mathrm{m})$ from paraffin-embedded blocks containing wounds were de-paraffinized and rehydrated with a graded series of alcohols. Slides were subjected to heat-induced antigen retrieval with $\mathrm{pH} 6.0$ citrate buffer. Slides were treated with primary antibodies against Ki-67 (Abcam, catalog no. ab16667, dilution 1:150), histone H4 (EMD Millipore, 05-858, 1:2000), CstF-64 (Bethyl Laboratories, IHC-00221, 1:1000), CPSF73 (Bethyl, A301-090A, 1:200) or CFIm25 (Sigma, AV40695, 1:200), followed by EnVision+ HRP-conjugated secondary antibody (Dako) and $\mathrm{DAB}$ chromogen (Roche) visualization. Slides were counterstained with hematoxylin and imaged with a Zeiss AXIO Imager.D2 microscope.

\section{Immunofluorescence}

A monolayer of contact-inhibited fibroblasts in a $35-\mathrm{mm}$ dish with a glass bottom (MatTek Corporation, Ashland, MA) was scratched (crosswise) using a sterile $1 \mathrm{ml}$ pipette tip to create a region free of cells (wound area). The cells were then gently washed two times using complete medium to remove the non-adherent cells generated during scratching. After $24 \mathrm{~h}$, the cells were fixed with $4 \%$ paraformaldehyde (Santa Cruz Biotechnology Inc., Dallas, TX) in PBS for $15 \mathrm{~min}$ at room temperature and then washed three times with ice-cold PBS. The cell permeabilization was performed using $0.25 \%$ Triton X-100 (Thermo Fisher Scientific, NJ) followed by washing the cells three times with PBS. The cells were blocked using blocking solution (1\% bovine serum album (BSA) in PBS containing 0.2\% Tween (Thermo Fisher Scientific) at room temperature for $30 \mathrm{~min}$. After blocking, the cells were incubated with primary antibodies (CstF64, CPSF73, or CFIm25) in blocking solution (1:100 dilution) at $4{ }^{\circ} \mathrm{C}$ in a humidified chamber overnight. The cells were then washed three times with PBS followed by incubation with Alexa-488 labeled secondary antibody (Thermo Fisher Scientific) at 1:250 dilution for $1 \mathrm{~h}$ at room temperature. After washing the cells three times with PBS, the cells were stained with DAPI using the VECTASHIELD hardset antifade mounting medium with DAPI (Vector Laboratories, Inc., Burlingame, CA). The images were taken at $10 \mathrm{X}$ magnification on a Zeiss confocal microscope (LSM 710, Carl Zeiss). Images were analyzed using ImageJ (v1.52a).

\section{siRNA transfection}

siRNAs against CFIm25 and CPSF73 were purchased from Sigma-Aldrich. siRNAs against CstF-64 were purchased from Sigma-Aldrich (CstF64.1) and Origene Technologies Inc., Rockville, MD (CstF64.2 and CstF64.3). siRNAs were transfected into fibroblasts or cancer cells using GeneMute transfection reagent from SignaGen Laboratories (Rockville, MD) according to the manufacturer's instructions.

\section{Real-time PCR}

For real-time PCR, DNA primers were designed with Primer3 for UBC primers or NCBI Primer-BLAST for all other primers, and synthesized by Integrated DNA Technologies (Coralville, IA). RNA was isolated using the PureLink RNA Kit (Thermo Fisher Scientific). cDNA was treated with TURBO DNA-free $e^{\text {Tw }}$ Kit (Thermo Fisher Scientific) to eliminate the remaining DNA. Real-time PCR was performed with $\mathrm{SYBR}^{\circ}$ Green One-Step Real-Time RT PCR Kit (Thermo Fisher Scientific). Samples were cycled on a BioRad CFX96 Real Time PCR instrument driving a Biorad C1000 Thermal Cycler for 40 cycles. The $\Delta \Delta C t$ method was used to determine the abundance of different PCR products [115]. Values for each gene of interest were normalized to UBC for the same sample. Primer sequences were as follows: CstF64, 5'-GCAAGCTTCTATGCAGGGT G-3' and 5' -TTGCATCGGCACTTGAACTC-3'; CPSF73, 5'-GAAGTCGAGGGGAGGAGTCT-3' and 5'-AGCTCC AAGGGGTCGGAT-3'; CFIm25, 5'-GCACCATCAACCT GTACCCTC-3' and 5' -AGTAACACATGGGGTAGCCG3'; long INF2, 5' -GGAGGAGGTGTGTGTCATCG-3' and 5' -CTCCTGCAGGGTTACTGGTG-3'; short INF2, 5'-G CTGCGGAACGAGTTTATCG-3' and 5'-GGAGGTGCT GCTTAGGTGAG-3'; long BOC, 5'-TCAGCAACGTGAT GATCTGTGA-3' and 5'-CCGCTCTATGGTTTCAGGA AGG-3'; short BOC 5'-CCTCATCTCTCCCACCCTGA A- ${ }^{\prime}$ ' and 5' $^{\prime}$-TGAGGTTTTCCAAGGGCACAA-3', UBC, $5^{\prime}$-TCTTGTTTGTGGATCGCTGTGA-3' and $5^{\prime}$-CAGG AGGGATGCCTTCCTTATC-3'.

\section{Incucyte in vitro wound healing assays}

For wound healing assays, fibroblasts were plated in the wells of an Incucyte ${ }^{\text {mix }}$ ImageLock $^{\text {Ti }}$ 96-well plate (Essen BioScience) and the WoundMaker ${ }^{\text {ra }}$ tool was used to create a denuded area in each well on the plate. The IncuCyte $^{\text {tw }}$ ZOOM live-cell analysis system (Essen BioScience) was used to automatically collect time-lapse images (phase-contrast) and to quantify cell migration over time as the density of cells in the denuded area relative to the density of cells out of the denuded area (relative wound density). Plots were determined to be statistically significantly different based on repeated measures two-way ANOVA with Dunnett's multiple comparison test. 


\section{Statistical analyses and plots}

Statistical significance determinations were performed with two-tailed tests for all analyses. For DESeq/DESeq2, splicing, and DEXSeq, the software included multiple hypothesis testing correction. All errors bars represent standard deviations. For the Wilcoxon test, we checked whether the data were normally distributed. We used Fisher's exact tests when sample sizes were low. Statistical significance for t-tests was determined using Prism (6.0f, GraphPad Software, La Jolla, CA). Statistical significance for correlations were performed using the cor() function in R. The hypergeometric test was performed with dhyper() function in R. The Wilcoxon test was performed with the Wilcox.test() function in R. Time series analysis for migration assays was performed with Prism. All bar graphs for RT-PCR and plots for migration assays were performed in Prism. All box plots and density plots were generated with ggplot2 package [116]. Plots for motif frequencies were generated in Prism.

\section{Additional files}

Additional file 1: Supplementary figures and supplementary Tables
S1-S5. (PDF $6052 \mathrm{~kb}$ )

Additional file 2: Expression of polyadenylation factors with quiescence. (XLSX $14 \mathrm{~kb}$ )

Additional file 3: Alternative isoform use with quiescence. (XLS $1401 \mathrm{~kb})$

Additional file 4: Gene Ontology for alternative isoform use. (XLSX $932 \mathrm{~kb}$ )

Additional file 5: Alternative splicing with quiescence. (XLSX $136 \mathrm{~kb}$ ) Additional file 6: Gene Ontology for alternative splicing. (XLSX 520 kb) Additional file 7: Polaydenylation site use with quiescence. (XLS 194 kb) Additional file 8: Polyadenylation site use with quiescence for genes with more than two polyadenylation sites. (XLSX 537 kb)

Additional file 9: Gene Ontology for alternative polyadenylation. (XLSX $618 \mathrm{~kb}$ )

Additional file 10: Alternative polaydenylation in knockdown cells. (XLS $309 \mathrm{~kb}$ )

Additional file 11: Differential expression with quiescence and knockdown. (XLSX $160 \mathrm{~kb}$ )

Additional file 12: Isoform-specific half-lives with quiescence. (XLSX $40 \mathrm{~kb}$ )

\section{Abbreviations}

7dCl: 7 days of contact inhibition; 7dSS: 7-day serum-starved;

APA: Alternative polyadenylation; BAM: Binary version of a SAM file; BCA: Bicinchoninic acid assay; BOC: Brother of CDO; CFIm25: Nudix (nucleoside diphosphate linked moiety X)-type motif 21; CLIP: Crosslinking immunoprecipitation; CLUAP1: Clusterin associated protein 1; CPSF: Cleavage and polyadenylation specificity factor; CSTF: Cleavage stimulation factor; CTD: Carboxy terminal domain; DAB: 3,3'-Diaminobenzidine; DMEM: Dulbecco's modified Eagle medium; FAK: Focal adhesion kinase; FBS: Fetal bovine serum; FDR: False discovery rate; FUS: Fused in sarcoma; GO: Gene ontology; GSEA: Gene set enrichment analysis; GSEMA: Gene Set Enrichment Made Awesome; HER2: Human epidermal growth factor receptor 2; HRP: Horse radish peroxidase; IGV: Integrated Genome Viewer; INF2 Inverted Formin: FH2 and WH2 domain containing; MEME: Multiple Em for Motif Elicitation; MPER: Mammalian protein extraction reagent; NMD: Nonsense-mediated decay; P: Proliferating; PAGE: Polyacrylamide gel electrophoresis; PPIH: Peptidylprolyl isomerase H; PRPF4: Pre-MRNA Processing Factor 4; rMATS: Replicate Multivariate Analysis of Transcript Splicing; RUD: Relative use of the distal polyadenylation site; SDS: Sodium dodecyl sulfate; TRA2B: Transformer-2 protein homolog beta; U1-70K: U1 small nuclear ribonucleoprotein 70K; U2AF2/U2AF65: U2 Small Nuclear RNA Auxiliary Factor 2; UBC: Ubiquitin C; UR APA: Upstream region APA or alternative polyadenylation affecting at least one polyadenylation site in the coding sequence; UTR APA: Alternative polyadenylation affecting polyadenylation sites in the UTR; WASp: Wiscott-Aldrich Syndrome protein

\section{Acknowledgements}

The authors acknowledge all of the members of the Coller laboratory, the hoffmann2 cluster, Jessica Buckles, Suhua Feng, and Marco Morselli for assistance.

\section{Funding}

HAC was the Milton E. Cassel scholar of the Rita Allen Foundation (http:// www.ritaallenfoundation.org). EU was supported in part by a National Science Foundation Graduate Research Fellowship DGE-0646086. This work was funded by Institute of General Medical Sciences Center of Excellence grant P50 GM071508, NIH R01 AR070245, PhRMA Foundation grant 2007RSGI9572, National Science Foundation Grant OCl-1047879 to David August, National Institute of General Medical Sciences R01 GM081686, National Institute of General Medical Sciences R01 GM0866465, the Eli \& Edythe Broad Center for Regenerative Medicine \& Stem Cell Research (Rose Hills and Hal Gaba awards), the Iris Cantor Women's Health Center/UCLA, the UCLA Clinical and Translational Science Institute Grant UL1TR000124, the Leukemia Lymphoma Society, the Melanoma Research Alliance 564714, NIH 1 R01-CA221296-01A1. AA was supported by a Broad Stem Cell Center fellowship, the Tumor Cell Biology Training Grant T32 CA009056 and a Dermatology Training Grant T32AR071307. $\mathrm{HAC}$ is a member of the Eli \& Edythe Broad Center of Regenerative Medicine \& Stem Cell Research, the Jonsson Comprehensive Cancer Center, the UCLA Molecular Biology Institute, and the UCLA Bioinformatics Interdepartmental Program. The funders had no role in study design, data collection and analysis, decision to publish, or preparation of the manuscript.

\section{Availability of data and materials}

The data that support this study are provided in supplementary tables. All the sequencing data are available at Gene Expression Omnibus data repository under the following accession numbers: GSE117444 (https:// www.ncbi.nlm.nih.gov/geo/query/acc.cgi?acc=GSE117444) [117], GSE117121 (https://www.ncbi.nlm.nih.gov/geo/query/acc.cgi?acc=GSE117121) [118], and GSE117033 (https://www.ncbi.nlm.nih.gov/geo/query/ acc.cgi?acc=GSE117033) [119].

\section{Authors' contributions}

MM contributed to study design, generating datasets, functional analysis, data analysis, and manuscript preparation. ELJ contributed to study design, generating datasets, data analysis, and manuscript preparation. VSS contributed to generating datasets and data analysis. LEN contributed to generating datasets and data analysis. DCC contributed to study design and generating datasets. DGR contributed to data analysis. DGT contributed to data analysis. AMA contributed to mouse wounding assays and data analysis. DJ contributed to mouse wounding assays and data analysis. SLB contributed to data analysis. HAC contributed to study design, data analysis and manuscript preparation. All authors read and approved the final manuscript.

\section{Ethics approval and consent to participate}

Human skin collection was approved by the Princeton University Institutional Review Board protocol number \#3134. Informed consent was obtained by the National Disease Research Interchange. Animal experimentation was approved by the UCLA Office for Animal Research, protocol number 2015033. All experimental methods comply with the Helsinki Declaration.

\section{Consent for publication}

Not applicable.

Competing interests

The authors declare that they have no competing interests. 


\section{Publisher's Note}

Springer Nature remains neutral with regard to jurisdictional claims in published maps and institutional affiliations.

\begin{abstract}
Author details
'Department of Molecular, Cell and Developmental Biology, University of California, Los Angeles, Los Angeles, CA, USA. ${ }^{2}$ Department of Biological Chemistry, David Geffen School of Medicine, University of California, Los Angeles, CA, USA. ${ }^{3}$ Department of Molecular Biology, Princeton University, Princeton, NJ, USA. ${ }^{4}$ Department of Biochemistry, University of California, Los Angeles, Los Angeles, CA, USA. ${ }^{5}$ Department of Chemical Engineering, University of California, Los Angeles, Los Angeles, CA, USA. ${ }^{6}$ Lewis-Sigler Institute for Integrative Genomics, Princeton University, Princeton, NJ, USA. ${ }^{7}$ Department of Computer Science, University of Southern California, Los Angeles, CA, USA.
\end{abstract}

Received: 17 May 2018 Accepted: 25 September 2018 Published online: 25 October 2018

\section{References}

1. Tschumperlin DJ. Fibroblasts and the ground they walk on. Physiology (Bethesda). 2013;28:380-90.

2. Hinz B, Phan SH, Thannickal VJ, Galli A, Bochaton-Piallat ML, Gabbiani G. The myofibroblast: one function, multiple origins. Am J Pathol. 2007;170:1807-16.

3. Werner S, Grose R. Regulation of wound healing by growth factors and cytokines. Physiol Rev. 2003;83:835-70.

4. De Donatis A, Ranaldi F, Cirri P. Reciprocal control of cell proliferation and migration. Cell Commun Signal. 2010;8:20.

5. Evertts AG, Manning AL, Wang X, Dyson NJ, Garcia BA, Coller HA. H4K20 methylation regulates quiescence and chromatin compaction. Mol Biol Cell. 2013;24:3025-37.

6. Suh EJ, Remillard MY, Legesse-Miller A, Johnson EL, Lemons JM, Chapman TR, Forman JJ, Kojima M, Silberman ES, Coller HA. A microRNA network regulates proliferative timing and extracellular matrix synthesis during cellular quiescence in fibroblasts. Genome Biol. 2012;13:R121.

7. Lemons JM, Feng XJ, Bennett BD, Legesse-Miller A, Johnson EL, Raitman I, Pollina EA, Rabitz HA, Rabinowitz JD, Coller HA. Quiescent fibroblasts exhibit high metabolic activity. PLoS Biol. 2010;8:e1000514.

8. Legesse-Miller A, Raitman I, Haley EM, Liao A, Sun LL, Wang DJ, Krishnan N, Lemons JM, Suh EJ, Johnson EL, et al. Quiescent fibroblasts are protected from proteasome inhibition-mediated toxicity. Mol Biol Cell. 2012;23:3566-81.

9. Coller HA, Sang L, Roberts JM. A new description of cellular quiescence. PLoS Biol. 2006;4:e83.

10. Iyer VR, Eisen MB, Ross DT, Schuler G, Moore T, Lee JC, Trent JM, Staudt LM, Hudson J Jr, Boguski MS, et al. The transcriptional program in the response of human fibroblasts to serum. Science. 1999;283:83-7.

11. Liu H, Adler AS, Segal E, Chang HY. A transcriptional program mediating entry into cellular quiescence. PLoS Genet. 2007;3:e91.

12. Sang $L$, Coller HA, Roberts JM. Control of the reversibility of cellular quiescence by the transcriptional repressor HES1. Science. 2008;321:1095-100.

13. Ji Z, Lee JY, Pan Z, Jiang B, Tian B. Progressive lengthening of $3^{\prime}$ untranslated regions of mRNAs by alternative polyadenylation during mouse embryonic development. Proc Natl Acad Sci U S A. 2009;106:7028-33.

14. Ji Z, Tian B. Reprogramming of 3' untranslated regions of mRNAs by alternative polyadenylation in generation of pluripotent stem cells from different cell types. PLoS One. 2009;4:e8419.

15. Hoque M, Ji Z, Zheng D, Luo W, Li W, You B, Park JY, Yehia G, Tian B. Analysis of alternative cleavage and polyadenylation by $3^{\prime}$ region extraction and deep sequencing. Nat Methods. 2013;10:133-9.

16. Tian B, Manley JL. Alternative polyadenylation of mRNA precursors. Nat Rev Mol Cell Biol. 2017;18(1):18-30. https://doi.org/10.1038/nrm.2016.116. Epub 2016 Sep 28.

17. Akman HB, Erson-Bensan AE. Alternative polyadenylation and its impact on cellular processes. Microrna. 2014;3:2-9.

18. Sandberg R, Neilson JR, Sarma A, Sharp PA, Burge CB. Proliferating cells express mRNAs with shortened $3^{\prime}$ untranslated regions and fewer microRNA target sites. Science. 2008;320:1643-7.

19. Takagaki Y, Seipelt RL, Peterson ML, Manley JL. The polyadenylation factor CstF-64 regulates alternative processing of IgM heavy chain pre-mRNA during B cell differentiation. Cell. 1996;87:941-52.
20. Mayr C, Bartel DP. Widespread shortening of 3'UTRs by alternative cleavage and polyadenylation activates oncogenes in cancer cells. Cell. 2009;138:673-84.

21. Elkon R, Drost J, van Haaften G, Jenal M, Schrier M, Vrielink JA, Agami R. E2F mediates enhanced alternative polyadenylation in proliferation. Genome Biol. 2012;13:R59.

22. Tian B, Manley JL. Alternative polyadenylation of mRNA precursors. Nat Rev Mol Cell Biol. 2017;18:18-30.

23. Shi Y, Manley JL. The end of the message: multiple protein-RNA interactions define the mRNA polyadenylation site. Genes Dev. 2015;29:889-97.

24. Legesse-Miller A, Elemento O, Pfau SJ, Forman JJ, Tavazoie S, Coller HA. Let7 overexpression leads to an increased fraction of cells in G2/M, direct down-regulation of $\mathrm{Cdc} 34$, and stabilization of Wee1 kinase in primary fibroblasts. J Biol Chem. 2009;284:6605-9.

25. Johnson EL, Wang W, Buckles J, Mitra M, Coller HA: Differential gene expression analysis between proliferating and quiescent human dermal fibroblasts. Data sets. GEO GSE117444. https://www.ncbi.nlm.nih.gov/geo/ query/acc.cgi?acc=GSE117444.

26. Venezia T, Merchant A, Ramos C, Whitehouse N, Young A, Shaw C, Goodell M. Molecular signatures of proliferation and quiescence in hematopoietic stem cells. PLoS Biol. 2004:2:e301.

27. Subramanian A, Tamayo P, Mootha VK, Mukherjee S, Ebert BL, Gillette MA, Paulovich A, Pomeroy SL, Golub TR, Lander ES, Mesirov JP. Gene set enrichment analysis: a knowledge-based approach for interpreting genomewide expression profiles. Proc Natl Acad Sci U S A. 2005;102:15545-50.

28. Mootha VK, Lindgren CM, Eriksson KF, Subramanian A, Sihag S, Lehar J, Puigserver P, Carlsson E, Ridderstrale M, Laurila E, et al. PGC-1alpharesponsive genes involved in oxidative phosphorylation are coordinately downregulated in human diabetes. Nat Genet. 2003;34:267-73.

29. Anders S, Reyes A, Huber W. Detecting differential usage of exons from RNA-seq data. Genome Res. 2012;22:2008-17.

30. Reimand J, Kull M, Peterson H, Hansen J, Vilo J. g: Profiler--a web-based toolset for functional profiling of gene lists from large-scale experiments. Nucleic Acids Res. 2007;35:W193-200.

31. Shen S, Park JW, Huang J, Dittmar KA, Lu ZX, Zhou Q, Carstens RP, Xing Y. MATS: a Bayesian framework for flexible detection of differential alternative splicing from RNA-Seq data. Nucleic Acids Res. 2012;40:e61.

32. Shen S, Park JW, Lu ZX, Lin L, Henry MD, Wu YN, Zhou Q, Xing Y. rMATS: robust and flexible detection of differential alternative splicing from replicate RNA-Seq data. Proc Natl Acad Sci U S A. 2014;111:E5593-601.

33. Park JW, Tokheim C, Shen S, Xing Y. Identifying differential alternative splicing events from RNA sequencing data using RNASeq-MATS. Methods Mol Biol. 2013;1038:171-9.

34. Lareau LF, Brenner SE. Regulation of splicing factors by alternative splicing and NMD is conserved between kingdoms yet evolutionarily flexible. Mol Biol Evol. 2015;32:1072-9.

35. Stoilov P, Daoud R, Nayler O, Stamm S. Human tra2-beta1 autoregulates its protein concentration by influencing alternative splicing of its pre-mRNA. Hum Mol Genet. 2004;13:509-24.

36. Anko ML, Muller-McNicoll M, Brandl H, Curk T, Gorup C, Henry I, Ule J, Neugebauer KM. The RNA-binding landscapes of two SR proteins reveal unique functions and binding to diverse RNA classes. Genome Biol. 2012;13:R17.

37. Jumaa H, Nielsen PJ. The splicing factor SRp20 modifies splicing of its own mRNA and ASF/SF2 antagonizes this regulation. EMBO J. 1997;16:5077-85.

38. Middleton R, Gao D, Thomas A, Singh B, Au A, Wong JJ, Bomane A, Cosson B, Eyras E, Rasko JE, Ritchie W. IRFinder: assessing the impact of intron retention on mammalian gene expression. Genome Biol. 2017;18:51.

39. Dichmann DS, Walentek P, Harland RM. The alternative splicing regulator Tra2b is required for somitogenesis and regulates splicing of an inhibitory Wnt11b isoform. Cell Rep. 2015;10:527-36.

40. Sibley CR. Regulation of gene expression through production of unstable mRNA isoforms. Biochem Soc Trans. 2014;42:1196-205.

41. Wong JJ, Ritchie W, Ebner OA, Selbach M, Wong JW, Huang Y, Gao D, Pinello N, Gonzalez M, Baidya K, et al. Orchestrated intron retention regulates normal granulocyte differentiation. Cell. 2013;154:583-95.

42. Sibley CR, Emmett W, Blazquez L, Faro A, Haberman N, Briese M, Trabzuni D, Ryten $M$, Weale ME, Hardy J, et al. Recursive splicing in long vertebrate genes. Nature. 2015;521:371-5.

43. Sakabe NJ, de Souza SJ. Sequence features responsible for intron retention in human. BMC Genomics. 2007:8:59. 
44. Lianoglou S, Garg V, Yang JL, Leslie CS, Mayr C. Ubiquitously transcribed genes use alternative polyadenylation to achieve tissue-specific expression. Genes Dev. 2013;27:2380-96.

45. Masamha CP, Xia Z, Yang J, Albrecht TR, Li M, Shyu AB, Li W, Wagner EJ. CFIm25 links alternative polyadenylation to glioblastoma tumour suppression. Nature. 2014;510:412-6.

46. Xia Z, Donehower LA, Cooper TA, Neilson JR, Wheeler DA, Wagner EJ, Li W. Dynamic analyses of alternative polyadenylation from RNA-seq reveal a 3'UTR landscape across seven tumour types. Nat Commun. 2014;5:5274.

47. Mitra M, Swamy VS, Wang W, Buckles J, Coller HA: Genome wide mapping of polyadenylation sites in proliferating and contact-inhibited cells and cells with knockdown of cleavage and polyadenylation factors. Data sets. GEO GSE117121. https://www.ncbi.nlm.nih.gov/geo/query/acc.cgi?acc=GSE117121.

48. Elkon R, Ugalde AP, Agami R. Alternative cleavage and polyadenylation: extent, regulation and function. Nat Rev Genet. 2013;14:496-506.

49. Beaudoing E, Freier S, Wyatt JR, Claverie JM, Gautheret D. Patterns of variant polyadenylation signal usage in human genes. Genome Res. 2000;10:1001-10.

50. Legendre $M$, Gautheret D. Sequence determinants in human polyadenylation site selection. BMC Genomics. 2003:4:7.

51. Millevoi S, Vagner S. Molecular mechanisms of eukaryotic pre-mRNA $3^{\prime}$ end processing regulation. Nucleic Acids Res. 2010;38:2757-74.

52. Shi Y, Di Giammartino DC, Taylor D, Sarkeshik A, Rice WJ, Yates JR 3rd, Frank J, Manley JL. Molecular architecture of the human pre-mRNA 3' processing complex. Mol Cell. 2009;33:365-76.

53. Tian B, Graber JH. Signals for pre-mRNA cleavage and polyadenylation. Wiley Interdiscip Rev RNA. 2012;3:385-96.

54. Brown KM, Gilmartin GM. A mechanism for the regulation of pre-mRNA 3' processing by human cleavage factor Im. Mol Cell. 2003;12:1467-76.

55. Mandel CR, Kaneko S, Zhang H, Gebauer D, Vethantham V, Manley JL, Tong L. Polyadenylation factor CPSF-73 is the pre-mRNA 3'-end-processing endonuclease. Nature. 2006:444:953-6.

56. Shell SA, Hesse C, Morris SM Jr, Milcarek C. Elevated levels of the 64-kDa cleavage stimulatory factor (CstF-64) in lipopolysaccharide-stimulated macrophages influence gene expression and induce alternative poly(A) site selection. J Biol Chem. 2005;280:39950-61.

57. Hwang HW, Park CY, Goodarzi H, Fak JJ, Mele A, Moore MJ, Saito Y, Darnell RB. PAPERCLIP identifies microRNA targets and a role of CstF64/64tau in promoting non-canonical poly(A) site usage. Cell Rep. 2016;15:423-35.

58. Gruber AR, Martin G, Keller W, Zavolan M. Cleavage factor Im is a key regulator of 3' UTR length. RNA Biol. 2012;9:1405-12.

59. Phatnani HP, Greenleaf AL. Phosphorylation and functions of the RNA polymerase II CTD. Genes Dev. 2006;20:2922-36.

60. Yao C, Choi EA, Weng L, Xie X, Wan J, Xing Y, Moresco JJ, Tu PG, Yates JR 3rd, Shi Y. Overlapping and distinct functions of CstF64 and CstF64tau in mammalian mRNA 3' processing. RNA. 2013;19:1781-90.

61. Li W, You B, Hoque M, Zheng D, Luo W, Ji Z, Park JY, Gunderson SI, Kalsotra A, Manley JL, Tian B. Systematic profiling of poly(a)+ transcripts modulated by core $3^{\prime}$ end processing and splicing factors reveals regulatory rules of alternative cleavage and polyadenylation. PLoS Genet. 2015;11:e1005166.

62. Mitra M, Nersesian LE, Wang W, Buckles J, Coller HA: To investigate the decay constants (half-lives) of transcript isoforms generated by alternative polyadenylation in proliferating and quiescent cells. Data sets. GEO GSE117121. https://www.ncbi.nlm.nih.gov/geo/query/acc.cgi?acc= GSE117121

63. Johnson EL, Robinson DG, Coller HA. Widespread changes in mRNA stability contribute to quiescence-specific gene expression patterns in a fibroblast model of quiescence. BMC Genomics. 2017:18:123.

64. Werner S, Krieg T, Smola H. Keratinocyte-fibroblast interactions in wound healing. J Invest Dermatol. 2007;127:998-1008.

65. Rakha EA, Ellis IO. Triple-negative/basal-like breast cancer: review. Pathology. 2009;41:40-7.

66. Erson-Bensan AE, Can T. Alternative polyadenylation: another foe in Cancer. Mol Cancer Res. 2016;14:507-17.

67. Shepard PJ, Choi EA, Lu J, Flanagan LA, Hertel KJ, Shi Y. Complex and dynamic landscape of RNA polyadenylation revealed by PAS-Seq. RNA. 2011;17:761-72

68. Singh P, Alley TL, Wright SM, Kamdar S, Schott W, Wilpan RY, Mills KD, Graber JH. Global changes in processing of mRNA 3' untranslated regions characterize clinically distinct cancer subtypes. Cancer Res. 2009;69:9422-30.

69. Graham RR, Kyogoku C, Sigurdsson S, Vlasova IA, Davies LR, Baechler EC, Plenge RM, Koeuth T, Ortmann WA, Hom G, et al. Three functional variants of IFN regulatory factor 5 (IRF5) define risk and protective haplotypes for human lupus. Proc Natl Acad Sci U S A. 2007;104:6758-63.

70. Kreth S, Limbeck E, Hinske LC, Schutz SV, Thon N, Hoefig K, Egensperger R, Kreth FW. In human glioblastomas transcript elongation by alternative polyadenylation and miRNA targeting is a potent mechanism of MGMT silencing. Acta Neuropathol. 2013;125:671-81.

71. Spies N, Burge CB, Bartel DP. 3' UTR-isoform choice has limited influence on the stability and translational efficiency of most mRNAs in mouse fibroblasts. Genome Res. 2013;23:2078-90.

72. Gruber AR, Martin G, Muller P, Schmidt A, Gruber AJ, Gumienny R, Mittal N, Jayachandran R, Pieters J, Keller W, et al. Global 3' UTR shortening has a limited effect on protein abundance in proliferating $T$ cells. Nat Commun. 2014:5:5465.

73. de Klerk E, Venema A, Anvar SY, Goeman JJ, Hu O, Trollet C, Dickson G, den Dunnen JT, van der Maarel SM, Raz V, t Hoen PA. Poly(a) binding protein nuclear 1 levels affect alternative polyadenylation. Nucleic Acids Res. 2012; 40:9089-101.

74. Gupta I, Clauder-Munster S, Klaus B, Jarvelin Al, Aiyar RS, Benes V, Wilkening S, Huber W, Pelechano V, Steinmetz LM. Alternative polyadenylation diversifies post-transcriptional regulation by selective RNA-protein interactions. Mol Syst Biol. 2014;10:719.

75. Geisberg JV, Moqtaderi Z, Fan X, Ozsolak F, Struhl K. Global analysis of mRNA isoform half-lives reveals stabilizing and destabilizing elements in yeast. Cell. 2014;156:812-24.

76. Protter DSW, Rao BS, Van Treeck B, Lin Y, Mizoue L, Rosen MK, Parker R. Intrinsically disordered regions can contribute promiscuous interactions to RNP granule assembly. Cell Rep. 2018;22:1401-12.

77. Fu Y, Sun Y, Li Y, Li J, Rao X, Chen C, Xu A. Differential genome-wide profiling of tandem 3' UTRs among human breast cancer and normal cells by high-throughput sequencing. Genome Res. 2011;21:741-7.

78. Lembo A, Di Cunto F, Provero P. Shortening of 3'UTRs correlates with poor prognosis in breast and lung cancer. PLoS One. 2012;7:e31129.

79. Aragaki M, Takahashi K, Akiyama H, Tsuchiya E, Kondo S, Nakamura Y, Daigo Y. Characterization of a cleavage stimulation factor, 3' pre-RNA, subunit 2,64 kDa (CSTF2) as a therapeutic target for lung cancer. Clin Cancer Res. 2011; 17:5889-900.

80. Jensen MA, Wilkinson JE, Krainer AR. Splicing factor SRSF6 promotes hyperplasia of sensitized skin. Nat Struct Mol Biol. 2014;21:189-97.

81. Wang ET, Cody NA, Jog S, Biancolella M, Wang TT, Treacy DJ, Luo S, Schroth GP, Housman DE, Reddy S, et al. Transcriptome-wide regulation of premRNA splicing and mRNA localization by muscleblind proteins. Cell. 2012; 150:710-24.

82. Sundaram GM, Common JE, Gopal FE, Srikanta S, Lakshman K, Lunny DP, Lim TC, Tanavde V, Lane EB, Sampath P. 'See-saw' expression of microRNA-198 and FSTL1 from a single transcript in wound healing. Nature. 2013;495:103-6.

83. Davis J, Salomonis N, Ghearing N, Lin SC, Kwong JQ, Mohan A, Swanson MS, Molkentin JD. MBNL1-mediated regulation of differentiation RNAs promotes myofibroblast transformation and the fibrotic response. Nat Commun. 2015;6:10084.

84. Long W, Yi P, Amazit L, LaMarca HL, Ashcroft F, Kumar R, Mancini MA, Tsai SY, Tsai MJ, O'Malley BW. SRC-3Delta4 mediates the interaction of EGFR with FAK to promote cell migration. Mol Cell. 2010;37:321-32.

85. Sieg DJ, Hauck CR, llic D, Klingbeil CK, Schaefer E, Damsky CH, Schlaepfer DD. FAK integrates growth-factor and integrin signals to promote cell migration. Nat Cell Biol. 2000;2:249-56.

86. Thrasher AJ. WASp in immune-system organization and function. Nat Rev Immunol. 2002;2:635-46.

87. Besson A, Gurian-West M, Schmidt A, Hall A, Roberts JM. p27Kip1 modulates cell migration through the regulation of RhoA activation. Genes Dev. 2004; 18:862-76.

88. Lawson CD, Burridge $K$. The on-off relationship of rho and Rac during integrin-mediated adhesion and cell migration. Small GTPases. 2014;5: e27958.

89. Lee HN, Mitra M, Bosompra O, Corney DC, Johnson EL, Rashed N, Ho LD, Coller HA. RECK isoforms have opposing effects on cell migration. Mol Biol Cell. 2018:mbcE17120708.

90. Pollina EA, Legesse-Miller A, Haley EM, Goodpaster T, Randolph-Habecker J, Coller HA. Regulating the angiogenic balance in tissues. Cell Cycle. 2008;7: 2056-70.

91. Mitra M, Ho LD, Coller HA. An in vitro model of cellular quiescence in primary human dermal fibroblasts. Methods Mol Biol. 2018;1686:27-47. 
92. Chomczynski P, Sacchi N. The single-step method of RNA isolation by acid guanidinium thiocyanate-phenol-chloroform extraction: twenty-something years on. Nat Protoc. 2006;1:581-5.

93. Chomczynski P, Sacchi N. Single-step method of RNA isolation by acid guanidinium thiocyanate phenol chloroform extraction. Anal Biochem. 1987; 162:156-9.

94. Trapnell C, Pachter L, Salzberg SL. TopHat: discovering splice junctions with RNA-Seq. Bioinformatics. 2009:25:1105-11.

95. Kim D, Pertea G, Trapnell C, Pimentel H, Kelley R, Salzberg SL. TopHat2: accurate alignment of transcriptomes in the presence of insertions, deletions and gene fusions. Genome Biol. 2013;14:R36.

96. Anders S, Huber W. Differential expression analysis for sequence count data. Genome Biol. 2010;11:R106.

97. Storey JD, Tibshirani R. Statistical significance for genomewide studies. Proc Natl Acad Sci U S A. 2003:100:9440-5.

98. Warnes GR, Bolker B, Bonebakker L, Gentleman R, Huber W, Liaw A, Lumley T, Maechler M, Magnusson A, Moeller S, et al: Gplots: various R programming tools for plotting data. R package version 2.14.2, $2014 \mathrm{http}: / /$ CRAN.R-project.org/package=gplots.

99. Team RC. R: a language and environment for statistical computing. Vienna: R Foundation for Statistical Computing; 2014.

100. Irizarry RA, Wang C, Zhou Y, Speed TP. Gene set enrichment analysis made simple. Stat Methods Med Res. 2009;18:565-75.

101. Robinson D: GSEAMA: gene set enrichment analysis made awesome. $R$ package version 0.99.0. 2014.http://github.com/dgrtwo/GSEAMA.

102. Picelli S, Faridani OR, Bjorklund AK, Winberg G, Sagasser S, Sandberg R. Fulllength RNA-seq from single cells using smart-seq2. Nat Protoc. 2014:9:171-81.

103. Gruber AJ, Schmidt R, Gruber AR, Martin G, Ghosh S, Belmadani M, Keller W, Zavolan M. A comprehensive analysis of $3^{\prime}$ end sequencing data sets reveals novel polyadenylation signals and the repressive role of heterogeneous ribonucleoprotein C on cleavage and polyadenylation. Genome Res Genome Res. 2016;26(8):1145-59. https://doi.org/10.1101/gr.202432.115. Epub 2016 Jul 5.

104. Love MI, Huber W, Anders S. Moderated estimation of fold change and dispersion for RNA-seq data with DESeq2. Genome Biol. 2014;15:550

105. Mitra M, Lee HN, Coller HA. Determining genome-wide transcript decay rates in proliferating and quiescent human fibroblasts. J Vis Exp. 2018(131) https://doi.org/10.3791/56423.

106. Bailey TL, Boden M, Buske FA, Frith M, Grant CE, Clementi L, Ren J, Li WW, Noble WS. MEME SUITE: tools for motif discovery and searching. Nucleic Acids Res. 2009;37:W202-8.

107. McLeay RC, Bailey TL. Motif enrichment analysis: a unified framework and an evaluation on ChIP data. BMC Bioinf. 2010;11:165.

108. Ray D, Kazan H, Cook KB, Weirauch MT, Najafabadi HS, Li X, Gueroussov S, Albu $\mathrm{M}$, Zheng $\mathrm{H}$, Yang $\mathrm{A}$, et al. A compendium of RNA-binding motifs for decoding gene regulation. Nature. 2013;499:172-7

109. Yao C, Biesinger J, Wan J, Weng L, Xing Y, Xie X, Shi Y. Transcriptome-wide analyses of CstF64-RNA interactions in global regulation of mRNA alternative polyadenylation. Proc Natl Acad Sci U S A. 2012;109:18773-8.

110. MacDonald CC, Wilusz J, Shenk T. The 64-kilodalton subunit of the CstF polyadenylation factor binds to pre-mRNAs downstream of the cleavage site and influences cleavage site location. Mol Cell Biol. 1994;14:6647-54.

111. Busch A, Hertel KJ. HEXEvent: a database of human EXon splicing events. Nucleic Acids Res. 2013;41:D118-24.

112. Pages $H$, Aoyoun P, Gentleman R, DebRoy S. Biostrings: string objects representing biological sequences, and matching algorithms. In: R package, version 2.40 .2 edition; 2016

113. Stojnic R, Diez D. PWMEnrich: PWM enrichment analysis. In: R package, version 4.8 .2 edition; 2015

114. Bembom O: seqLogo: sequence logos for DNA sequence alignments. Vol. R package, version 1.38 .0 edition; 2016.

115. Livak KJ, Schmittgen TD. Analysis of relative gene expression data using real-time quantitative PCR and the 2(-Delta Delta C(T)) method. Methods. 2001;25:402-8.

116. Wickam H. ggplot2: elegant graphics for data analysis. New York: SpringerVerlag ed; 2009

117. Mitra M, Johnson EL, Swamy VS, Nersesian LE, Corney DC, Robinson DG, Taylor DG, Ambrus AM, Jelinek D, Wang W, Batista SL, Coller HA. Alternative polyadenylation factors link cell cycle to migration. Gene expression omnibus.2018 https://www.ncbi.nlm.nih.gov/geo/query/acc.cgi?acc= GSE117444.
118. Mitra M, Johnson EL, Swamy VS, Nersesian LE, Corney DC, Robinson DG, Taylor DG, Ambrus AM, Jelinek D, Wang W, Batista SL, Coller HA. Alternative polyadenylation factors link cell cycle to migration. Gene expression omnibus. 2018 https://www.ncbi.nlm.nih.gov/geo/query/acc.cgi?acc= GSE117121.

119. Mitra M, Johnson EL, Swamy VS, Nersesian LE, Corney DC, Robinson DG, Taylor DG, Ambrus AM, Jelinek D, Wang W, Batista SL, Coller HA. Alternative polyadenylation factors link cell cycle to migration. Gene expression omnibus 2018. https://www.ncbi.nlm.nih.gov/geo/query/acc.cgi?acc= GSE117033.

120. Schneider TD, Stephens RM. Sequence logos: a new way to display consensus sequences. Nucleic Acids Res. 1990;18:6097-100.

121. Schneider TD, Stormo GD, Gold L, Ehrenfeucht A. Information content of binding sites on nucleotide sequences. J Mol Biol. 1986;188:415-31.

122. Tsurusawa M, Fujimoto T. Cell cycle progression and phenotypic modification of Ki67 antigen-negative G1- and G2-phase cells in phorbol ester-treated Molt-4 human leukemia cells. Cytometry. 1995;20:146-53.
Ready to submit your research? Choose BMC and benefit from:

- fast, convenient online submission

- thorough peer review by experienced researchers in your field

- rapid publication on acceptance

- support for research data, including large and complex data types

- gold Open Access which fosters wider collaboration and increased citations

- maximum visibility for your research: over $100 \mathrm{M}$ website views per year

At $\mathrm{BMC}$, research is always in progress.

Learn more biomedcentral.com/submissions 